\title{
PERTURBATION ANALYSIS ON MATRIX PENCILS FOR TWO SPECIFIED EIGENPAIRS OF A SEMISIMPLE EIGENVALUE WITH MULTIPLICITY TWO*
}

\author{
SK. SAFIQUE AHMAD ${ }^{\dagger}$ AND PRINCE KANHYA ${ }^{\ddagger}$
}

\begin{abstract}
In this paper, we derive backward error formulas of two approximate eigenpairs of a semisimple eigenvalue with multiplicity two for structured and unstructured matrix pencils. We also construct the minimal structured perturbations with respect to the Frobenius norm such that these approximate eigenpairs become exact eigenpairs of an appropriately perturbed matrix pencil. The structures we consider include T-symmetric/T-skewsymmetric, Hermitian/skew-Hermitian, T-even/T-odd, and H-even/H-odd matrix pencils. Further, we establish various relationships between the backward error of a single approximate eigenpair and the backward error of two approximate eigenpairs of a semisimple eigenvalue with multiplicity two.
\end{abstract}

Key words. multiple eigenvalue, semisimple eigenvalue, defective eigenvalue, structured generalized eigenvalue problem, eigenpair backward error

AMS subject classifications. 65F15, 15A18, 65F 35, 15A12

1. Introduction. In the process of obtaining eigenvalues and eigenvectors of a given matrix pencil by using numerical methods, we encounter different situations of obtained eigenpairs/eigenvalues. For example, the obtained eigenvalue can be simple, semisimple, and repeating but defective. These different situations arise because of the diverse nature of the obtained eigenvectors. Further, these obtained eigenpairs/eigenvalues are approximate, not exact; this happens due to the roundoff errors. Different authors have developed the backward error analysis for a single approximate eigenpair/eigenvalue to understand how accurate the computed solution is for the matrix pencil and to check if this computed solution is useful or not. Backward error analysis plays an important role in understanding the accuracy of the computed solutions; it provides the minimum perturbation in some appropriate norm such that given approximate eigenpairs/eigenvalues become exact for an appropriately perturbed problem. Malyshev [15] has discussed the minimal perturbation of a given $n$-by- $n$ matrix to the nearest matrices that have $\lambda \in \mathbb{C}$ as a multiple eigenvalue with respect to the 2-norm. Further, this work has been extended for two distinct prescribed numbers, and the nearest matrix has been obtained that contains these prescribed numbers in its spectrum; see [8, 13, 17]. For a given $n$-by- $n$ matrix, the above work has been extended for $k(k \leq n)$ prescribed eigenvalues by Lippert [14] and Kokabifar et al. [10]. For the matrix polynomial setup, E. Kokabifara et al. [11] have extended the above idea to $k$ specified distinct eigenvalues and provided the backward error and the minimum perturbed matrix polynomial for the unstructured case. Similar to the backward error of eigenvalues, different authors have developed the backward error analysis of a single approximate eigenpair for unstructured as well as structured matrix pencils and matrix polynomials; see $[1,4,5,2,12,19]$.

For the matrix case, Tisseur [21] has extended the backward error results from one specified eigenpair to more specified eigenpairs. The author has obtained the backward error formula for Hermitian, skew-Hermitian, complex symmetric, complex skew-symmetric and doubly structured matrices using [20, Lemma 1.4] and [21, Lemma 2.4], along with

${ }^{*}$ Received December 8, 2019. Accepted April 13, 2020. Published online on August 25, 2020. Recommended by Froilan Dopico. The research work of S. S. Ahmad is funded by SERB, DST, India, Grant Number: SR/FTP/MS043/2011. The research work of P. Kanhaya is funded by SERB Grant Number: SR/FTP/MS-043/2011 and through the grant UGC-Ref.No.:947(CSIR-UGC NET DEC. 2018).

${ }^{\dagger}$ Discipline of Mathematics, Indian Institute of Technology Indore, Simrol, Indore-453552, Madhya Pradesh, India (safiqueliiti.ac.in, safique@gmail.com).

${ }^{\ddagger}$ Research Scholar Discipline of Mathematics, IIT Indore, India (pkanhya@gmail . com). 
the "W-trick"1. Tisseur has investigated the structured backward error analysis by imposing appropriate conditions on approximate eigenpairs, for example, while computing the backward error result for Hermitian matrices, the author has assumed that the columns of $X_{k}$, the approximate eigenvectors matrix, are orthonormal. This condition seems to be natural as we always get a set of orthonormal vectors for a given Hermitian matrix. Similar to the Hermitian case, the author has imposed two natural conditions during the backward error analysis of Hermitian unitary matrices: the first one is the orthonormality condition on $X_{k}$, and the second one is the approximate eigenvalues matrix $\Lambda_{k}=\operatorname{diag}( \pm 1)$. In the same manner, we will discuss natural conditions on the given approximate eigenpairs to perform the backward error analysis. Next, in [7] Chu and Golub have studied the backward error analysis of one or more approximate eigenpairs for unstructured nonsquare matrix pencils when the approximate eigenvalues are distinct and the eigenvectors are linearly independent. Though they considered one or more eigenpairs and obtained the unstructured backward error, results of the backward error analysis of more than one approximate eigenpairs for structured matrix pencils are still unanswered.

The above discussion on the backward error analysis of approximate eigenvalues/eigenpairs for unstructured/structured matrices, matrix pencils, and matrix polynomials leads to a natural question: what will be the cumulative backward error of two approximate eigenpairs of a given matrix pencil? Before finding the answer to this question, we want to emphasize the point that whenever the author in [21] has imposed a condition on $X_{k}$ or $\Lambda_{k}$ to obtain the structured backward error formula, that condition seems to be a natural one for that particular structure. In a similar manner to answer the above-raised question, we will find out natural condition(s) on approximate eigenpairs under which we can obtain the backward error results for a large class of matrix pencils.

To understand the natural condition, we recall one important result: if an eigenvalue of a matrix pencil is repeating but semisimple, we always get a set of orthonormal eigenvectors corresponding to that eigenvalue; see Lemma 2.5 for more information. Using this result, we obtain the backward error formula for two approximate eigenpairs of a semisimple eigenvalue with multiplicity two. Here we add that a generic situation for a multiple eigenvalue is a double eigenvalue; see, for example, [16]. For obtaining backward error results, we adopt and extend the technique of $[1,4,5]$. This technique is based on the orthonormal properties of approximate eigenvectors. In general, we can not get orthonormal vectors corresponding to distinct eigenvalues, hence the question of finding the structured backward error of two approximate eigenpairs is still open when the eigenvalues are distinct or defective. Though in this paper, we obtain structured backward error results for the matrix pencil setup, one can also derive the results of [21] for Hermitian, skew-Hermitian, real symmetric, and real skew-symmetric matrices for the semisimple case by using our technique. In this manuscript, we answer the above raised question for structured as well as unstructured matrix pencils. We work with T-symmetric/T-skew-symmetric, Hermitian/skew-Hermitian, T-even/T-odd, and $H$-even/H-odd matrix pencils; see $[6,9,18,24]$ for more on structured matrix pencils and matrix polynomials.

Let $\mathbf{L}\left(\mathbb{C}^{n \times n}\right)$ be the space of matrix pencils, and let $\mathbf{L} \in \mathbf{L}\left(\mathbb{C}^{n \times n}\right)$ be of the form $\mathbf{L}(\alpha):=$ $\alpha_{0} A_{0}+\alpha_{1} A_{1}$, where $A_{0}, A_{1} \in \mathbb{C}^{n \times n}, \alpha=\left(\alpha_{0}, \alpha_{1}\right) \in \mathbb{C}^{2}$. Suppose that $\left(\lambda, x_{1}\right)$ and $\left(\lambda, x_{2}\right)$ are two approximate eigenpairs of $\mathbf{L}$, where $\lambda \in \mathbb{C}^{2} \backslash\{(0,0)\}$ and $0 \neq x_{i} \in \mathbb{C}^{n}, i=1,2$. In this work, we find the nearest $\delta \mathbf{L} \in \mathbf{L}\left(\mathbb{C}^{n \times n}\right)$ of the form $\delta \mathbf{L}(\alpha):=\alpha_{0} \delta A_{0}+\alpha_{1} \delta A_{1}$, where $\delta A_{0}, \delta A_{1} \in \mathbb{C}^{n \times n}$ are such that two approximate eigenpairs $\left(\lambda, x_{1}\right)$ and $\left(\lambda, x_{2}\right)$ become the exact eigenpairs of $\mathbf{L}+\delta \mathbf{L}$. We use the Frobenius norm to investigate the structured backward error analysis. Results are developed in such a way that T-symmetric/T-skew-symmetric cases

${ }^{1}$ See $[21$, Section 3.2] for more information on the W-trick. 
are presented in a single framework. Similarly, Hermitian/skew-Hermitian, T-even/T-odd, and $\mathrm{H}$-even/H-odd cases are also presented in a single framework. Further, we find relationships between the backward error of a single approximate eigenpair, the backward error of two approximate eigenpairs for a semisimple eigenvalue with multiplicity two, and the structured backward error of two approximate eigenpairs for a semisimple eigenvalue with multiplicity two.

This paper is organized as follows. In Section 2, we provide notation and preliminary results. We generalize the definition of a single approximate eigenpair to more than one approximate eigenpairs for structured and unstructured matrix pencils. Section 3 deals with the backward error analysis of $T$-symmetric/T-skew-symmetric matrix pencils. A relationship between existing and developed backward errors is also discussed in that section. In Section 4, we obtain the backward error result for unstructured matrix pencils. We also establish a relationship between structured and unstructured backward errors. Similarly, in Section 5, we discuss the perturbation analysis of Hermitian/skew-Hermitian matrix pencils. Further, backward error results for the $H$-even/H-odd and T-even/T-odd cases are discussed in Sections 6 and 7 , respectively. At the end of Section 7, we present a table summarizing the various relations between unstructured and structured backward errors of a single and two approximate eigenpairs. We illustrate our theory by an example in Section 8, and the conclusions are given in Section 9.

2. Notation and preliminaries. Let $\mathbb{R}$ and $\mathbb{C}$ be the sets of real and complex numbers, respectively. Throughout this paper, $\mathbb{C}^{n \times m}$ is the set of all $n \times m$ complex matrices. For $A \in \mathbb{C}^{n \times m}, A^{T}$ and $A^{H}$ denote the transpose and conjugate transpose of $A$, respectively. The Frobenius norm of $A \in \mathbb{C}^{n \times m}$ is defined as $\|A\|_{F}:=\sqrt{\operatorname{trace}\left(A^{H} A\right)}$. For $x_{1}, x_{2} \in \mathbb{C}^{n}$, define

$$
P_{x_{1}: x_{2}}:=\left(I-x_{1} x_{1}^{H}-x_{2} x_{2}^{H}\right), \quad P_{x_{1}}^{c}:=\left(I-x_{2} x_{2}^{H}\right), \quad P_{x_{2}}^{c}:=\left(I-x_{1} x_{1}^{H}\right) .
$$

For $x \in \mathbb{C}^{n}, \bar{x}$ denotes the conjugate of $x$. For $z \in \mathbb{C}$, the real and imaginary parts of $z$ are denoted by $\Re(z)$ and $\Im(z)$, respectively. Let $\mathbf{L}\left(\mathbb{C}^{n \times n}\right)$ be the space of matrix pencils. Then a homogeneous matrix pencil $\mathbf{L} \in \mathbf{L}\left(\mathbb{C}^{n \times n}\right)$ is defined as :

$$
\mathbf{L}(\alpha):=\alpha_{0} A_{0}+\alpha_{1} A_{1}, \quad A_{0}, A_{1} \in \mathbb{C}^{n \times n}, \alpha=\left(\alpha_{0}, \alpha_{1}\right) \in \mathbb{C}^{2} .
$$

Finding $\lambda=\left(\lambda_{0}, \lambda_{1}\right) \in \mathbb{C}^{2} \backslash\{(0,0)\}, 0 \neq x \in \mathbb{C}^{n}$, such that $\mathbf{L}(\lambda) x=0$, is called the generalized eigenvalue problem, $\lambda$ is called an eigenvalue of (2.1), and $x$ is the corresponding right eigenvector. If $0 \neq y \in \mathbb{C}^{n}$ such that $y^{H} \mathbf{L}(\lambda)=0$, then $y$ is called the left eigenvector corresponding to $\lambda$. We denote the matrix pencil defined in (2.1) by $\mathbf{L}$, and $(\lambda, x)$ is called an eigenpair of $\mathbf{L}$. We define

$$
\|\mathbf{L}\|_{F}:=\left\|\left(\left\|A_{0}\right\|_{F},\left\|A_{1}\right\|_{F}\right)\right\|_{2}=\left(\left\|A_{0}\right\|_{F}^{2}+\left\|A_{1}\right\|_{F}^{2}\right)^{1 / 2},
$$

where $\|\cdot\|_{F}$ denotes the Frobenius norm on $\mathbb{C}^{n \times n}$, and $\|\cdot\|_{2}$ denotes the 2-norm on $\mathbb{C}^{n}$. Non-homogeneous matrix pencils can be obtain by fixing $\alpha_{0}=1$ in (2.1).

DEFINITION 2.1. A matrix pencil $\mathbf{L} \in \mathbf{L}\left(\mathbb{C}^{n \times n}\right)$ of the form (2.1) is said to be regular if $\operatorname{det}(\mathbf{L}(\lambda)) \neq 0$ for some $\lambda=\left(\lambda_{0}, \lambda_{1}\right) \in \mathbb{C}^{2} \backslash\{(0,0)\}$. Otherwise, it is called a singular matrix pencil.

We denote the spectrum of $\mathbf{L}$ by $\Lambda(\mathbf{L})$, and it is given by

$$
\Lambda(\mathbf{L}):=\left\{\lambda=\left(\lambda_{0}, \lambda_{1}\right) \in \mathbb{C}^{2} \backslash\{(0,0)\}: \operatorname{det}(\mathbf{L}(\lambda))=0\right\} .
$$

If $\left(\lambda_{0}, \lambda_{1}\right) \in \mathbb{C}^{2} \backslash\{(0,0)\}$ is an eigenvalue of $\mathbf{L}$, then $\left(a \lambda_{0}, a \lambda_{1}\right)$ is another representation of the eigenvalue $\left(\lambda_{0}, \lambda_{1}\right)$ for any $0 \neq a \in \mathbb{C}$. Hence for a given homogeneous $\mathbf{L}$, we normalize 
$\left(\lambda_{0}, \lambda_{1}\right) \in \Lambda(\mathbf{L})$ by $\left|\lambda_{0}\right|^{2}+\left|\lambda_{1}\right|^{2}=1$ and consider $\Lambda(\mathbf{L})$ is a subset of the unit sphere $\mathbf{S}^{1}:=\left\{\left(\lambda_{0}, \lambda_{1}\right) \in \mathbb{C}^{2}:\left|\lambda_{0}\right|^{2}+\left|\lambda_{1}\right|^{2}=1\right\}$. By working in a homogeneous setup, one can handle the infinity eigenvalue together with the finite eigenvalue; see [3] for more details on homogeneous eigenvalue problems. Throughout this paper, we consider regular matrix pencils for the establishment of our results.

DEFINITION 2.2. The algebraic multiplicity (A.M.) of an eigenvalue $\lambda=\left(\lambda_{0}, \lambda_{1}\right) \in$ $\Lambda(\mathbf{L})$ is its multiplicity as a zero of the characteristic polynomial $\operatorname{det}(\mathbf{L}(\lambda))$. The geometric multiplicity (G.M.) of an eigenvalue $\left(\lambda_{0}, \lambda_{1}\right) \in \Lambda(\mathbf{L})$ is defined as the dimension of the subspace $\operatorname{ker}(\mathbf{L}(\lambda))$. An eigenvalue is said to be semisimple if its algebraic multiplicity is equal to its geometric multiplicity; see [23] for more details on semisimple eigenvalues.

Let $\mathbf{L}$ be a matrix pencil of the form (2.1), and let $\lambda=\left(\lambda_{0}, \lambda_{1}\right) \in \mathbb{C}^{2} \backslash\{(0,0)\}$ be its eigenvalue. Then $\lambda$ is said to be a double eigenvalue if its algebraic multiplicity is two. We will consider a double-semisimple eigenvalue for the backward error analysis since a generic situation for a multiple eigenvalue is a double eigenvalue; see $[16,22]$ for more information on double-semisimple eigenvalues. We work with structured matrix pencils of the form (2.1). These structured matrix pencils are defined in Table 2.1 based on the properties of the matrices $A_{0}, A_{1} \in \mathbb{C}^{n \times n}$.

TABLE 2.1

Different structured matrix pencils

\begin{tabular}{l|ll}
$\mathbf{S}$ & \multicolumn{2}{|l}{ Matrix structure } \\
\hline T-symmetric & $A_{0}=A_{0}^{T}$, & $A_{1}=A_{1}^{T}$ \\
T-skew-symmetric & $A_{0}=-A_{0}^{T}$, & $A_{1}=-A_{1}^{T}$ \\
Hermitian & $A_{0}=A_{0}^{H}$, & $A_{1}=A_{1}^{H}$ \\
skew-Hermitian & $A_{0}=-A_{0}^{H}$, & $A_{1}=-A_{1}^{H}$ \\
T-even & $A_{0}=A_{0}^{T}$, & $A_{1}=-A_{1}^{T}$ \\
T-odd & $A_{0}=-A_{0}^{T}$, & $A_{1}=A_{1}^{T}$ \\
H-even & $A_{0}=A_{0}^{H}$, & $A_{1}=-A_{1}^{H}$ \\
$H$-odd & $A_{0}=-A_{0}^{H}$, & $A_{1}=A_{1}^{H}$
\end{tabular}

In the following, we extend the backward error defined for a single approximate eigenpair to the case of two approximate eigenpairs; the backward error analysis for a single approximate eigenpair has been discussed in [1].

DEFINITION 2.3. Let $\mathbf{L}$ be a matrix pencil of the form (2.1). Let $\left(\lambda, x_{1}\right)$ and $\left(\lambda, x_{2}\right)$ be two approximate eigenpairs of $\mathbf{L}$, where $\lambda \in \mathbb{C}^{2} \backslash\{(0,0)\}$ and $0 \neq x_{1}, x_{2} \in \mathbb{C}^{n}$. Then the unstructured and structured backward errors of two approximate eigenpairs $\left(\lambda, x_{1}\right)$ and $\left(\lambda, x_{2}\right)$ are defined by

$$
\begin{aligned}
& \eta_{F}\left(\lambda, x_{1}, x_{2}, \mathbf{L}\right):=\inf \left\{\|\delta \mathbf{L}\|_{F} \mid(\mathbf{L}(\lambda)+\delta \mathbf{L}(\lambda)) x_{i}=0, \text { for } i=1,2\right\}, \\
& \eta_{F}^{\mathbf{S}}\left(\lambda, x_{1}, x_{2}, \mathbf{L}\right):=\inf \left\{\|\delta \mathbf{L}\|_{F} \mid \delta \mathbf{L} \in \mathbf{S}, \quad(\mathbf{L}(\lambda)+\delta \mathbf{L}(\lambda)) x_{i}=0, \text { for } i=1,2\right\},
\end{aligned}
$$

respectively. Here $\delta \mathbf{L}$ is a matrix pencil of the form (2.1) with $\delta \mathbf{L}(\alpha)=\alpha_{0} \delta A_{0}+\alpha_{1} \delta A_{1}$, where $\delta A_{0}, \delta A_{1} \in \mathbb{C}^{n \times n},\|\delta \mathbf{L}\|_{F}:=\sqrt{\left\|\delta A_{0}\right\|_{F}^{2}+\left\|\delta A_{1}\right\|_{F}^{2}}$, and

$$
\begin{gathered}
\mathbf{S} \in\{T \text {-symmetric, } T \text {-skew-symmetric, Hermitian, skew-Hermitian, } \\
T \text {-even, } T \text {-odd, } H \text {-even, } H \text {-odd }\} .
\end{gathered}
$$

Now we recall some results useful in this paper. 
REMARK 2.4. The eigenvectors corresponding to a double-semisimple eigenvalue of a matrix pencil $\mathbf{L}$, are not uniquely determined. Using this information, we will establish the following lemma.

LEMMA 2.5. Suppose that $p=\left(p_{0}, p_{1}\right) \in \mathbb{C}^{2} \backslash\{(0,0)\}$ is a double-semisimple eigenvalue of a matrix pencil $\mathbf{L}$. Then there exist two orthonormal vectors $y_{1}, y_{2} \in \mathbb{C}^{n}$, such that $\mathbf{L}(p) y_{i}=0$, for $i=1,2$. In particular, every double-semisimple eigenvalue $p$ of $\mathbf{L}$ has two orthonormal eigenvectors.

Proof. Let $\left(p_{0}, p_{1}\right)$ be a double-semisimple eigenvalue of $\mathbf{L}$. This implies that its algebraic and geometric multiplicity will be two; hence there exist two linearly independent eigenvectors $z_{1}, z_{2} \in \mathbb{C}^{n}$, such that $\mathbf{L}(p) z_{i}=0$, for $i=1,2$. By the Gram-Schmidt process, we can set $y_{1}=z_{1}$ and $y_{2}=z_{2}-\gamma z_{1}$, where $\gamma=\frac{z_{1}^{H} z_{2}}{z_{1}^{H} z_{1}} \in \mathbb{C}$. We can easily see that $\mathbf{L}(p) y_{i}=0$, and $y_{1}, y_{2}$ are orthogonal, and in particular orthonormal.

The above lemma guarantees that for a double-semisimple eigenvalue, we always have two orthonormal eigenvectors.

REMARK 2.6. Using the Gram-Schmidt process, we can extend the above lemma for a semisimple eigenvalue with algebraic multiplicity more than two.

After recalling these preliminary results, we establish in the following section backward error results for two eigenpairs of a double-semisimple eigenvalue.

REMARK 2.7. Since we are interested in this article in finding the backward error of two approximate eigenpairs of a double-semisimple eigenvalue, in light of Lemma 2.5, from now on we will take the orthonormal eigenvectors corresponding to a double-semisimple eigenvalue.

3. Backward error for $\boldsymbol{T}$-symmetric/T-skew-symmetric matrix pencils. In this section, we present the structured backward error analysis of two approximate eigenpairs of a double-semisimple eigenvalue for T-symmetric/T-skew-symmetric matrix pencils. We start this section with the following existence theorem. Throughout this section, $\epsilon=1$ represents a $T$-symmetric matrix pencil and $\epsilon=-1$ represents a $T$-skew-symmetric matrix pencil.

THEOREM 3.1. Let $\mathbf{L} \in \mathbf{L}\left(\mathbb{C}^{n \times n}\right)$ be a T-symmetric/T-skew-symmetric homogeneous matrix pencil of the form (2.1). Let $\left(\lambda, x_{1}\right)$ and $\left(\lambda, x_{2}\right)$ be two approximate eigenpairs of $\mathbf{L}$, where $\lambda=\left(\lambda_{0}, \lambda_{1}\right) \in \mathbb{C}^{2} \backslash\{(0,0)\}$ is a double-semisimple eigenvalue and $x_{1}, x_{2} \in \mathbb{C}^{n}$ are orthonormal vectors. Set $k_{i}:=-\mathbf{L}(\lambda) x_{i}$, for $i=1,2$, and define

$$
\delta A_{0}=\sum_{i=1}^{2} \bar{\lambda}_{0} \frac{k_{i} x_{i}^{H}+\epsilon \overline{x_{i}} k_{i}^{T} P_{x_{1}: x_{2}}}{H_{2}^{2}(\lambda)} \quad \text { and } \quad \delta A_{1}=\sum_{i=1}^{2} \bar{\lambda}_{1} \frac{k_{i} x_{i}^{H}+\epsilon \overline{x_{i}} k_{i}^{T} P_{x_{1}: x_{2}}}{H_{2}^{2}(\lambda)}
$$

where $H_{2}(\lambda)=\left(\left|\lambda_{0}\right|^{2}+\left|\lambda_{1}\right|^{2}\right)^{1 / 2}$. Then there exists a T-symmetric/T-skew-symmetric matrix pencil $\delta \mathbf{L} \in \mathbf{L}\left(\mathbb{C}^{n \times n}\right)$ of the form $\delta \mathbf{L}(\alpha)=\alpha_{0} \delta A_{0}+\alpha_{1} \delta A_{1}$, such that $(\mathbf{L}(\lambda)+\delta \mathbf{L}(\lambda)) x_{i}=0$, for $i=1,2$.

Proof. The proof follows from some simple calculations.

LEMMA 3.2. Let $\mathbf{L} \in \mathbf{L}\left(\mathbb{C}^{n \times n}\right)$ be a T-symmetric/T-skew-symmetric homogeneous matrix pencil of the form (2.1). Let $\left(\lambda, x_{1}\right)$ and $\left(\lambda, x_{2}\right)$ be two approximate eigenpairs of $\mathbf{L}$, where $\lambda=\left(\lambda_{0}, \lambda_{1}\right) \in \mathbb{C}^{2} \backslash\{(0,0)\}$ is a double-semisimple eigenvalue and $x_{1}, x_{2} \in \mathbb{C}^{n}$ are orthonormal vectors. Set $k_{i}:=-\mathbf{L}(\lambda) x_{i}$, for $i=1,2$. Then the following equality holds for $t=1,2$,

$$
\left(x_{1}^{T} k_{2}\right)\left(\epsilon \bar{x}_{2} x_{1}^{H}+\bar{x}_{1} x_{2}^{H}\right) x_{t}=\sum_{j=1, j \neq t}^{2} \bar{x}_{j} x_{j}^{T} k_{t}
$$



$x_{2}^{T} k_{1}$.

Proof. The proof follows, with some simple calculations, from the fact that $\epsilon x_{1}^{T} k_{2}=$

Next, we establish the main result of this section.

THEOREM 3.3. Let $\mathbf{L} \in \mathbf{L}\left(\mathbb{C}^{n \times n}\right)$ be a T-symmetric/T-skew-symmetric homogeneous matrix pencil of the form (2.1). Let $\left(\lambda, x_{1}\right)$ and $\left(\lambda, x_{2}\right)$ be two approximate eigenpairs of $\mathbf{L}$, where $\lambda=\left(\lambda_{0}, \lambda_{1}\right) \in \mathbb{C}^{2} \backslash\{(0,0)\}$ is a double-semisimple eigenvalue and $x_{1}, x_{2} \in \mathbb{C}^{n}$ are orthonormal vectors. Set $k_{i}:=-\mathbf{L}(\lambda) x_{i}$, for $i=1,2$. Then there exists a $T$-symmetric/Tskew-symmetric $\delta \mathbf{L}$ of the form $\delta \mathbf{L}(\alpha)=\alpha_{0} \delta A_{0}+\alpha_{1} \delta A_{1}$, such that $(\mathbf{L}(\lambda)+\delta \mathbf{L}(\lambda)) x_{i}=0$. The perturbation matrices are given by

$$
\begin{aligned}
\delta A_{0} & =\sum_{i=1}^{2}\left(\bar{\lambda}_{0} \frac{\bar{P}_{x_{i}}^{c} k_{i} x_{i}^{H}+\epsilon \overline{x_{i}} k_{i}^{T} P_{x_{1}: x_{2}}}{H_{2}^{2}(\lambda)}\right)+\frac{\bar{\lambda}_{0}\left(x_{1}^{T} k_{2}\right)\left(\epsilon \bar{x}_{2} x_{1}^{H}+\bar{x}_{1} x_{2}^{H}\right)}{H_{2}^{2}(\lambda)}, \\
\delta A_{1} & =\sum_{i=1}^{2}\left(\bar{\lambda}_{1} \frac{\bar{P}_{x_{i}}^{c} k_{i} x_{i}^{H}+\epsilon \overline{x_{i}} k_{i}^{T} P_{x_{1}: x_{2}}}{H_{2}^{2}(\lambda)}\right)+\frac{\bar{\lambda}_{1}\left(x_{1}^{T} k_{2}\right)\left(\epsilon \bar{x}_{2} x_{1}^{H}+\bar{x}_{1} x_{2}^{H}\right)}{H_{2}^{2}(\lambda)} .
\end{aligned}
$$

The backward error is given by

$$
\left(\eta_{F}^{\mathbf{S}}\left(\lambda, x_{1}, x_{2}, \mathbf{L}\right)\right)^{2}=\sum_{i=1}^{2}\left(\frac{2\left\|k_{i}\right\|_{2}^{2}-\frac{(1+\epsilon)}{2}\left|x_{i}^{T} k_{i}\right|^{2}}{H_{2}^{2}(\lambda)}\right)-2 \frac{\left|x_{2}^{T} k_{1}\right|^{2}}{H_{2}^{2}(\lambda)} .
$$

Proof. From Theorem 3.1, there exists a $T$-symmetric/T-skew-symmetric $\delta \mathbf{L}$ of the form $\delta \mathbf{L}(\alpha)=\alpha_{0} \delta A_{0}+\alpha_{1} \delta A_{1}$, such that $\mathbf{L}(\lambda) x_{i}+\delta \mathbf{L}(\lambda) x_{i}=0$, for $i=1,2$. To construct $\delta A_{j}$, $j=0,1$, such that $\delta A_{j}=\epsilon \delta A_{j}^{T}$, we consider

$$
\widetilde{\delta A_{j}}=U^{T} \delta A_{j} U=\underset{n-2}{2}\left[\begin{array}{c|c}
2 & n-2 \\
\hline \delta A_{j} & \epsilon \delta B_{j}^{T} \\
\hline \delta B_{j} & \delta D_{j}
\end{array}\right]
$$

where

$$
\widehat{\delta A_{j}}=\left[\begin{array}{cc}
\frac{(1+\epsilon)}{2} \delta a_{j, 11} & \epsilon \delta a_{j, 12} \\
\delta a_{j, 12} & \frac{(1+\epsilon)}{2} \delta a_{j, 22}
\end{array}\right], \quad \delta B_{j}=\left[\begin{array}{ll}
b_{j 1} & b_{j 2}
\end{array}\right], \quad \delta D_{j}=\epsilon \delta D_{j}{ }^{T},
$$

for $j=0,1$, and $U \in \mathbb{C}^{n \times n}$ is a unitary matrix such that $U=\left[\begin{array}{ll}V_{1} & V_{2}\end{array}\right]$ with $V_{1}=\left[\begin{array}{ll}x_{1} & x_{2}\end{array}\right]$ $\in \mathbb{C}^{n \times 2}$.

We need to construct $\delta \mathbf{L}$, such that $(\mathbf{L}(\lambda)+\delta \mathbf{L}(\lambda)) x_{i}=0$, for $i=1$, 2. Since it is given that $k_{i}=-\mathbf{L}(\lambda) x_{i}$, we get $k_{i}=\delta \mathbf{L}(\lambda) x_{i}$. From (3.1), we get $\widetilde{\delta} \mathbf{L}(\lambda)=U^{T} \delta \mathbf{L}(\lambda) U$. Using the properties of $U$, we get $\widetilde{\delta \mathbf{L}}(\lambda) U^{H} x_{i}=U^{T} \delta \mathbf{L}(\lambda) x_{i}=U^{T} k_{i}$. This implies

$$
\lambda_{0}\left[\begin{array}{cc}
\widehat{\delta A_{0}} & \epsilon \delta B_{0}^{T} \\
\delta B_{0} & \delta D_{0}
\end{array}\right]\left[\begin{array}{c}
e_{i} \\
0
\end{array}\right]+\lambda_{1}\left[\begin{array}{cc}
\widehat{\delta A_{1}} & \epsilon \delta B_{1}^{T} \\
\delta B_{1} & \delta D_{1}
\end{array}\right]\left[\begin{array}{c}
e_{i} \\
0
\end{array}\right]=\left[\begin{array}{c}
V_{1}^{T} k_{i} \\
V_{2}^{T} k_{i}
\end{array}\right],
$$

and further simplification gives

$$
\left[\begin{array}{c}
\left(\lambda_{0} \widehat{\delta A_{0}}+\lambda_{1} \widehat{\delta A_{1}}\right) e_{i} \\
\left(\lambda_{0} \delta B_{0}+\lambda_{1} \delta B_{1}\right) e_{i}
\end{array}\right]=\left[\begin{array}{l}
V_{1}^{T} k_{i} \\
V_{2}^{T} k_{i}
\end{array}\right]
$$

where $e_{i} \in \mathbb{C}^{2}$ is a vector having 1 at the $i^{t h}$ position and 0 elsewhere. From (3.2), we get the following equations

$$
\begin{array}{rr}
\frac{(1+\epsilon)}{2} \lambda_{0} \delta a_{0, i i}+\frac{(1+\epsilon)}{2} \lambda_{1} \delta a_{1, i i}=x_{i}^{T} k_{i}, & i=1,2, \\
\lambda_{0} b_{0 i}+\lambda_{1} b_{1 i}=V_{2}^{T} k_{i}, & i=1,2 .
\end{array}
$$


The minimum norm solutions of (3.3) and (3.4) are given by

$$
\begin{aligned}
\delta a_{0, i i} & =\frac{(1+\epsilon)}{2} \frac{\bar{\lambda}_{0}}{H_{2}^{2}(\lambda)} x_{i}^{T} k_{i}, & \delta a_{1, i i} & =\frac{(1+\epsilon)}{2} \frac{\bar{\lambda}_{1}}{H_{2}^{2}(\lambda)} x_{i}^{T} k_{i}, \\
b_{0 i} & =\frac{\bar{\lambda}_{0}}{H_{2}^{2}(\lambda)} V_{2}^{T} k_{i}, & b_{1 i} & =\frac{\bar{\lambda}_{1}}{H_{2}^{2}(\lambda)} V_{2}^{T} k_{i} .
\end{aligned}
$$

By (3.2), we get two more equations

$$
\begin{gathered}
\lambda_{0} \delta a_{0,12}+\lambda_{1} \delta a_{1,12}=x_{2}^{T} k_{1}, \\
\lambda_{0} \delta a_{0,12}+\lambda_{1} \delta a_{1,12}=\epsilon x_{1}^{T} k_{2} .
\end{gathered}
$$

Since $A_{j}=\epsilon A_{j}^{T}$, for $j=0,1$, we obtain $\epsilon x_{1}^{T} k_{2}=x_{2}^{T} k_{1}$; hence equations (3.5) and (3.6) are identical. The minimum norm solution of (3.6) is given by $\delta a_{0,12}=\frac{\epsilon \bar{\lambda}_{0}}{H_{2}^{2}(\lambda)} x_{1}^{T} k_{2}, \delta a_{1,12}=$ $\frac{\epsilon \bar{\lambda}_{1}}{H_{2}^{2}(\lambda)} x_{1}^{T} k_{2}$. Substituting back all the obtained entries into (3.1), we get

$$
\delta A_{j}=\bar{U}\left[\begin{array}{ccc}
\frac{(1+\epsilon)}{2} \frac{\bar{\lambda}_{j}}{H_{2}^{2}(\lambda)} x_{1}^{T} k_{1} & \frac{\bar{\lambda}_{j}}{H_{2}^{2}(\lambda)} x_{1}^{T} k_{2} & \epsilon \frac{\bar{\lambda}_{j}}{H_{2}^{2}(\lambda)}\left(V_{2}^{T} k_{1}\right)^{T} \\
\frac{\epsilon \bar{\lambda}_{j}}{H_{2}^{2}(\lambda)} x_{1}^{T} k_{2} & \frac{(1+\epsilon)}{2} \frac{\lambda_{j}}{H_{2}^{2}(\lambda)} x_{2}^{T} k_{2} & \epsilon \frac{\bar{\lambda}_{j}}{H_{2}^{2}(\lambda)}\left(V_{2}^{T} k_{2}\right)^{T} \\
\frac{\bar{\lambda}_{j}}{H_{2}^{2}(\lambda)} V_{2}^{T} k_{1} & \frac{\bar{\lambda}_{j}}{H_{2}^{2}(\lambda)} V_{2}^{T} k_{2} & \delta D_{j}
\end{array}\right] U^{H} .
$$

Further simplifying (3.7) and setting $\delta D_{j}=0$, we get the desired structured perturbation matrices $\delta A_{0}$ and $\delta A_{1}$ whose Frobenius norms are minimal. For $i=1,2$, we need to show that $\left((\mathbf{L}(\lambda)+\delta \mathbf{L}(\lambda)) x_{i}=0\right.$. Consider

$$
\begin{aligned}
(\mathbf{L}(\lambda)+\delta \mathbf{L}(\lambda)) x_{i} & =\mathbf{L}(\lambda) x_{i}+\delta \mathbf{L}(\lambda) x_{i}=-k_{i}+\lambda_{0} \delta A_{0} x_{i}+\lambda_{1} \delta A_{1} x_{i} \\
& =-k_{i}+\bar{P}_{x_{i}}^{c} k_{i}+\left(x_{1}^{T} k_{2}\right)\left(\epsilon \bar{x}_{2} x_{1}^{H}+\bar{x}_{1} x_{2}^{H}\right) x_{i} .
\end{aligned}
$$

Using Lemma 3.2, we get $(\mathbf{L}(\lambda)+\delta \mathbf{L}(\lambda)) x_{i}=-k_{i}+\bar{P}_{x_{i}}^{c} k_{i}+\sum_{j=1, j \neq i}^{2} \bar{x}_{j} x_{j}^{T} k_{i}=0$.

Since the Frobenius norms of $\delta A_{0}$ and $\delta A_{1}$ are minimal, we find that

$$
\left(\eta_{F}^{\mathbf{S}}\left(\lambda, x_{1}, x_{2}, \mathbf{L}\right)\right)^{2}=\left\|\delta A_{0}\right\|_{F}^{2}+\left\|\delta A_{1}\right\|_{F}^{2},
$$

where

$$
\begin{aligned}
& \left\|\delta A_{0}\right\|_{F}^{2}+\left\|\delta A_{1}\right\|_{F}^{2}=\sum_{j=0}^{1}\left\|\widehat{\delta A_{j}}\right\|_{F}^{2}+\left(1+\epsilon^{2}\right)\left\|\delta B_{j}\right\|_{F}^{2} \\
& =\sum_{j=0}^{1}\left\|\widehat{\delta A_{j}}\right\|_{F}^{2}+2\left\|\delta B_{j}\right\|_{F}^{2}=\sum_{i=1}^{2} \frac{(1+\epsilon)}{2} \frac{\left|x_{i}^{T} k_{i}\right|^{2}}{H_{2}^{2}(\lambda)}+2 \frac{\left|x_{1}^{T} k_{2}\right|^{2}}{H_{2}^{2}(\lambda)}+2 \frac{\left\|V_{2}^{T} k_{i}\right\|^{2}}{H_{2}^{2}\left(\lambda_{i}\right)} .
\end{aligned}
$$

Since $\left\|V_{2}^{T} k_{i}\right\|^{2}=\left\|k_{i}\right\|^{2}-\left|x_{1}^{T} k_{i}\right|^{2}-\left|x_{2}^{T} k_{i}\right|^{2}$ and using Remark 3.6, we get

$$
\left(\eta_{F}^{\mathbf{S}}\left(\lambda, x_{1}, x_{2}, \mathbf{L}\right)\right)^{2}=\sum_{i=1}^{2}\left(\frac{2\left\|k_{i}\right\|_{2}^{2}-\frac{(1+\epsilon)}{2}\left|x_{i}^{T} k_{i}\right|^{2}}{H_{2}^{2}(\lambda)}\right)-2 \frac{\left|x_{2}^{T} k_{1}\right|^{2}}{H_{2}^{2}(\lambda)} .
$$

REMARK 3.4. Results for non-homogeneous matrix pencils can be obtained by fixing $\lambda_{0}=1$ in Theorem 3.3.

REMARK 3.5. By extending $\left\{x_{1}, x_{2}\right\}$ to a basis of $\mathbb{C}^{n}$, we get another $(n-2)$ linearly independent vectors $\left\{x_{3}, \ldots, x_{n}\right\}$. Then using the Gram-Schmidt process for $\left\{x_{1}, \ldots, x_{n}\right\}$, we obtain the desired $V_{2} \in \mathbb{C}^{n \times(n-2)}$. 
REMARK 3.6. For $\epsilon=1,-1$, we have $\epsilon x_{1}^{T} k_{2}=x_{2}^{T} k_{1}$, and $\left|\epsilon x_{1}^{T} k_{2}\right|^{2}=\left|x_{1}^{T} k_{2}\right|^{2}=$ $\left|x_{2}^{T} k_{1}\right|^{2}$.

COROLlary 3.7. Let $\mathbf{L}$ be a non-homogeneous T-symmetric/T-skew-symmetric nonhomogeneous matrix pencil of the form $\mathbf{L}(\gamma)=A_{0}+\gamma A_{1}$. Let $\left(\mu, x_{1}\right)$ and $\left(\mu, x_{2}\right)$ be two approximate eigenpairs, such that $\mu \in \mathbb{C}$ is a double-semisimple eigenvalue and $x_{1}, x_{2} \in \mathbb{C}^{n}$ are orthonormal vectors. Set $k_{i}:=-\mathbf{L}(\mu) x_{i}$, for $i=1,2$. Then the following holds:

$$
\left(\eta_{F}^{\mathbf{S}}\left(\mu, x_{1}, x_{2}, \mathbf{L}\right)\right) \leq \sqrt{\eta_{F}^{\mathbf{S}}\left(\mu, x_{1}, \mathbf{L}\right)^{2}+\eta_{F}^{\mathbf{S}}\left(\mu, x_{2}, \mathbf{L}\right)^{2}} .
$$

Proof. For the T-symmetric case, by substituting $\lambda_{0}=1, \lambda_{1}=\mu$, and $\epsilon=1$ in Theorem 3.3, we get the following relation:

$$
\left(\eta_{F}^{\mathbf{S}}\left(\mu, x_{1}, x_{2}, \mathbf{L}\right)\right)^{2}=\sum_{i=1}^{2}\left(\frac{2\left\|k_{i}\right\|_{2}^{2}-\left|x_{i}^{T} k_{i}\right|^{2}}{\left(1+|\mu|^{2}\right)}\right)-2 \frac{\left|x_{2}^{T} k_{1}\right|^{2}}{\left(1+|\mu|^{2}\right)} .
$$

From [1, Theorem 3.1], we have

$$
\left(\eta_{F}^{\mathbf{S}}\left(\mu, x_{i}, \mathbf{L}\right)\right)^{2}=\frac{2\left\|k_{i}\right\|_{2}^{2}-\left|x_{i}^{T} k_{i}\right|^{2}}{\left(1+|\mu|^{2}\right)}, \quad i=1,2 .
$$

By substituting equation (3.9) in (3.8), we get

$$
\left(\eta_{F}^{\mathbf{S}}\left(\mu, x_{1}, x_{2}, \mathbf{L}\right)\right)^{2}=\sum_{i=1}^{2}\left(\eta_{F}^{\mathbf{S}}\left(\mu, x_{i}, \mathbf{L}\right)\right)^{2}-2 \frac{\left|x_{2}^{T} k_{1}\right|^{2}}{\left(1+|\mu|^{2}\right)} .
$$

Since $\frac{\left|x_{2}^{T} k_{1}\right|^{2}}{\left(1+|\mu|^{2}\right)} \geq 0$, we get the desired result.

REMARK 3.8. The result for the T-skew-symmetric case can be obtained in a similar manner by using $\epsilon=-1$ and [1, Theorem 3.2].

Next, we present the backward error analysis for unstructured matrix pencils, and by that analysis, we will establish a relationship between structured and unstructured backward errors.

4. Backward error analysis for unstructured matrix pencils. In this section, we derive the backward error formula for two approximate eigenpairs of a double-semisimple eigenvalue without imposing any structure on the matrix pencils. We start this section with the following theorem, which guarantees that there always exists a matrix pencil for two approximate eigenpairs of a double-semisimple eigenvalue.

THEOREM 4.1. Let $\mathbf{L} \in \mathbf{L}\left(\mathbb{C}^{n \times n}\right)$ be a matrix pencil of the form (2.1). Let $\left(\lambda, x_{1}\right)$ and $\left(\lambda, x_{2}\right)$ be two approximate eigenpairs of $\mathbf{L}$, where $\lambda=\left(\lambda_{0}, \lambda_{1}\right) \in \mathbb{C}^{2} \backslash\{(0,0)\}$ is a double-semisimple eigenvalue and $x_{1}, x_{2} \in \mathbb{C}^{n}$ are orthonormal vectors. Set $k_{i}:=-\mathbf{L}(\lambda) x_{i}$, for $i=1,2$, and define

$$
\delta A_{0}=\sum_{i=1}^{2} \bar{\lambda}_{0} \frac{k_{i} x_{i}^{H}+\bar{x}_{i} x_{i}^{T} P_{x_{1}: x_{2}}}{H_{2}^{2}(\lambda)} \quad \text { and } \quad \delta A_{1}=\sum_{i=1}^{2} \bar{\lambda}_{1} \frac{k_{i} x_{i}^{H}+\bar{x}_{i} x_{i}^{T} P_{x_{1}: x_{2}}}{H_{2}^{2}(\lambda)}
$$

where $H_{2}(\lambda)=\left(\left|\lambda_{0}\right|^{2}+\left|\lambda_{1}\right|^{2}\right)^{1 / 2}$. Then there exists a matrix pencil $\delta \mathbf{L} \in \mathbf{L}\left(\mathbb{C}^{n \times n}\right)$ of the form $\delta \mathbf{L}(\alpha)=\alpha_{0} \delta A_{0}+\alpha_{1} \delta A_{1}$, such that $(\mathbf{L}(\lambda)+\delta \mathbf{L}(\lambda)) x_{i}=0$, for $i=1,2$.

Proof. The proof follows from some simple calculations.

Now we present the main result of this section.

THEOREM 4.2. Let $\mathbf{L} \in \mathbf{L}\left(\mathbb{C}^{n \times n}\right)$ be a homogeneous matrix pencil of the form (2.1). Let $\left(\lambda, x_{1}\right)$ and $\left(\lambda, x_{2}\right)$ be two approximate eigenpairs of $\mathbf{L}$, where $\lambda=\left(\lambda_{0}, \lambda_{1}\right) \in \mathbb{C}^{2} \backslash\{(0,0)\}$ 
is a double-semisimple eigenvalue and $x_{1}, x_{2} \in \mathbb{C}^{n}$ are orthonormal vectors. Set $k_{i}:=$ $-\mathbf{L}(\lambda) x_{i}$, for $i=1,2$. Then there exists a matrix pencil $\delta \mathbf{L}$ of the form $\delta \mathbf{L}(\alpha)=\alpha_{0} \delta A_{0}+$ $\alpha_{1} \delta A_{1}$, such that $(\mathbf{L}(\lambda)+\delta \mathbf{L}(\lambda)) x_{i}=0$. The perturbation matrices are given by

$$
\delta A_{0}=\sum_{i=1}^{2} \frac{\bar{\lambda}_{0} k_{i} x_{i}^{H}}{H_{2}^{2}(\lambda)}, \quad \delta A_{1}=\sum_{i=1}^{2} \frac{\bar{\lambda}_{1} k_{i} x_{i}^{H}}{H_{2}^{2}(\lambda)} .
$$

The unstructured backward error is given by

$$
\left(\eta_{F}\left(\lambda, x_{1}, x_{2}, \mathbf{L}\right)\right)^{2}=\sum_{i=1}^{2} \frac{\left\|k_{i}\right\|_{2}^{2}}{H_{2}^{2}(\lambda)} .
$$

Proof. From Theorem 4.1, there always exists a matrix pencil $\delta \mathbf{L}$ of the form $\delta \mathbf{L}(\alpha)=$ $\alpha_{0} \delta A_{0}+\alpha_{1} \delta A_{1}$, such that $\mathbf{L}(\lambda) x_{i}+\delta \mathbf{L}(\lambda) x_{i}=0$, for $i=1,2$. To construct $\delta A_{j}$ for $j=0,1$, we consider

$$
\widetilde{\delta A_{j}}=U^{T} \delta A_{j} U={ }_{n-2}^{2}\left[\begin{array}{c|c}
\widehat{\delta A_{j}} & \delta C_{j}^{T} \\
\hline \delta B_{j} & \delta D_{j}
\end{array}\right]
$$

where

$$
\widehat{\delta A_{j}}=\left[\begin{array}{ll}
\delta a_{j, 11} & \delta a_{j, 12} \\
\delta a_{j, 21} & \delta a_{j, 22}
\end{array}\right], \quad \delta B_{j}=\left[\begin{array}{ll}
b_{j 1} & b_{j 2}
\end{array}\right], \quad \delta C_{j}=\left[\begin{array}{ll}
c_{j 1} & c_{j 2}
\end{array}\right],
$$

for $j=0,1$, and $U \in \mathbb{C}^{n \times n}$ is a unitary matrix such that $U=\left[\begin{array}{ll}V_{1} & V_{2}\end{array}\right]$ with $V_{1}=\left[\begin{array}{ll}x_{1} & x_{2}\end{array}\right]$ $\in \mathbb{C}^{n \times 2}$. Since we need to construct $\delta \mathbf{L}$, such that $(\mathbf{L}(\lambda)+\delta \mathbf{L}(\lambda)) x_{i}=0$, we get $k_{i}=$ $\delta \mathbf{L}(\lambda) x_{i}$, for $i=1,2$. From $\widetilde{\delta} \mathbf{L}(\lambda)=U^{T} \delta \mathbf{L}(\lambda) U$, we have $\widetilde{\delta} \mathbf{L}(\lambda) U^{H} x_{i}=U^{T} \delta \mathbf{L}(\lambda) x_{i}=$ $U^{T} k_{i}$. This implies

$$
\lambda_{0}\left[\begin{array}{ll}
\widehat{\delta A_{0}} & \delta C_{0}^{T} \\
\delta B_{0} & \delta D_{0}
\end{array}\right]\left[\begin{array}{c}
e_{i} \\
\mathbf{0}
\end{array}\right]+\lambda_{1}\left[\begin{array}{cc}
\widehat{\delta A_{1}} & \delta C_{1}^{T} \\
\delta B_{1} & \delta D_{1}
\end{array}\right]\left[\begin{array}{c}
e_{i} \\
\mathbf{0}
\end{array}\right]=\left[\begin{array}{c}
V_{1}^{T} k_{i} \\
V_{2}^{T} k_{i}
\end{array}\right],
$$

and further simplifications give

$$
\left[\begin{array}{c}
\left(\lambda_{0} \widehat{\delta A_{0}}+\lambda_{1} \widehat{\delta A_{1}}\right) e_{i} \\
\left(\lambda_{0} \delta B_{0}+\lambda_{1} \delta B_{1}\right) e_{i}
\end{array}\right]=\left[\begin{array}{c}
V_{1}^{T} k_{i} \\
V_{2}^{T} k_{i}
\end{array}\right]
$$

where $e_{i} \in \mathbb{C}^{2}$ is a vector having 1 at $i^{t h}$ position and 0 elsewhere. From (4.2), we get the following equations

$$
\begin{array}{rr}
\lambda_{0} \delta a_{0, i i}+\lambda_{1} \delta a_{1, i i}=x_{i}^{T} k_{i}, & i=1,2 \\
\lambda_{0} b_{0 i}+\lambda_{1} b_{1 i}=V_{2}^{T} k_{i}, & i=1,2
\end{array}
$$

The minimum norm solutions of (4.3) and (4.4) are given by

$$
\delta a_{0, i i}=\frac{\bar{\lambda}_{0}}{H_{2}^{2}(\lambda)} x_{i}^{T} k_{i}, \quad \delta a_{1, i i}=\frac{\bar{\lambda}_{1}}{H_{2}^{2}(\lambda)} x_{i}^{T} k_{i}, \quad b_{0 i}=\frac{\bar{\lambda}_{0}}{H_{2}^{2}(\lambda)} V_{2}^{T} k_{i}, \quad b_{1 i}=\frac{\bar{\lambda}_{1}}{H_{2}^{2}(\lambda)} V_{2}^{T} k_{i} .
$$

Further from (4.2), we get the following two equations:

$$
\begin{gathered}
\lambda_{0} \delta a_{0,21}+\lambda_{1} \delta a_{1,21}=x_{2}^{T} k_{1}, \\
\lambda_{0} \delta a_{0,12}+\lambda_{1} \delta a_{1,12}=x_{1}^{T} k_{2} .
\end{gathered}
$$


The minimum norm solutions of (4.5) and (4.6) are given by

$$
\begin{aligned}
\delta a_{0,21} & =\frac{\bar{\lambda}_{0}}{H_{2}^{2}(\lambda)} x_{2}^{T} k_{1}, & \delta a_{1,21} & =\frac{\bar{\lambda}_{1}}{H_{2}^{2}(\lambda)} x_{2}^{T} k_{1}, \\
\delta a_{0,12} & =\frac{\bar{\lambda}_{0}}{H_{2}^{2}(\lambda)} x_{1}^{T} k_{2}, & \delta a_{1,12} & =\frac{\bar{\lambda}_{1}}{H_{2}^{2}(\lambda)} x_{1}^{T} k_{2} .
\end{aligned}
$$

Similar to the T-symmetric $T$-skew-symmetric cases, substituting back all these obtained entries into (4.1) along with $\delta D_{1}=\delta D_{2}=0$, and $\delta C_{1}=\delta C_{2}=0$, we get the desired perturbation matrices with minimal Frobenius norms. Similar to Theorem 3.3, we can obtain the backward error for the unstructured case, which is given by

$$
\eta_{F}\left(\lambda, x_{1}, x_{2}, \mathbf{L}\right)=\sqrt{\sum_{i=1}^{2} \frac{\left\|k_{i}\right\|_{2}^{2}}{H_{2}^{2}(\lambda)}} .
$$

After establishing the unstructured backward error formula for two approximate eigenpairs, we now establish a relationship between unstructured and $T$-symmetric $T$-skew-symmetric backward errors.

COROLlaRY 4.3. Let $\mathbf{L} \in \mathbf{L}\left(\mathbb{C}^{n \times n}\right)$ be a T-symmetric/ T-skew-symmetric matrix pencil of the form (2.1). Let $\left(\lambda, x_{1}\right)$ and $\left(\lambda, x_{2}\right)$ be two approximate eigenpairs of $\mathbf{L}$, where $x_{1}, x_{2} \in \mathbb{C}^{n}$ are orthonormal vectors and $\lambda=\left(\lambda_{0}, \lambda_{1}\right) \in \mathbb{C}^{2} \backslash\{(0,0)\}$ is a double-semisimple eigenvalue. Then the following holds:

$$
\left(\eta_{F}^{\mathbf{S}}\left(\lambda, x_{1}, x_{2}, \mathbf{L}\right)\right) \leq \sqrt{2}\left(\eta_{F}\left(\lambda, x_{1}, x_{2}, \mathbf{L}\right)\right) .
$$

Proof. From Theorem 3.3, we get $\left(\eta_{F}^{\mathbf{S}}\left(\lambda, x_{1}, x_{2}, \mathbf{L}\right)\right)^{2} \leq \sum_{i=1}^{2} \frac{2\left\|k_{i}\right\|_{2}^{2}}{H_{2}^{2}(\lambda)}$. Also using Theorem 4.2, we have $\left(\eta_{F}\left(\lambda, x_{1}, x_{2}, \mathbf{L}\right)\right)^{2}=\sum_{i=1}^{2} \frac{\left\|k_{i}\right\|_{2}^{2}}{H_{2}^{2}(\lambda)}$. Hence we get

$$
\left(\eta_{F}^{\mathbf{S}}\left(\lambda, x_{1}, x_{2}, \mathbf{L}\right)\right) \leq \sqrt{2}\left(\eta_{F}\left(\lambda, x_{1}, x_{2}, \mathbf{L}\right)\right)
$$

Now we present a relationship between the backward error of a single eigenpair and the backward error of two approximate eigenpairs of a double-semisimple eigenvalue.

COROLLARY 4.4. Let $\left(\mu, x_{1}\right)$ and $\left(\mu, x_{2}\right)$ be two approximate eigenpairs, such that $\mu \in \mathbb{C}$ is a double-semisimple eigenvalue of a non-homogeneous matrix pencil $\mathbf{L}$ of the form $\mathbf{L}(\gamma)=A_{0}+\gamma A_{1}$. Set $k_{i}:=-\mathbf{L}(\mu) x_{i}$, where $x_{i} \in \mathbb{C}^{n}$, for $i=1,2$. Then the following holds:

$$
\eta_{F}\left(\mu, x_{1}, x_{2}, \mathbf{L}\right)=\sqrt{\eta_{F}^{2}\left(\mu, x_{1}, \mathbf{L}\right)+\eta_{F}^{2}\left(\mu, x_{2}, \mathbf{L}\right)} .
$$

Proof. By substituting $\lambda_{0}=1$ and $\lambda_{1}=\mu$ in Theorem 4.2, we get

$$
\eta_{F}\left(\mu, x_{1}, x_{2}, \mathbf{L}\right)=\sqrt{\sum_{i=1}^{2} \frac{\left\|k_{i}\right\|_{2}^{2}}{\left(1+|\mu|^{2}\right)}} .
$$

On the other hand, by [1], we have $\eta_{F}\left(\mu, x_{i}, \mathbf{L}\right)=\frac{\left\|k_{i}\right\|}{\left(1+|\mu|^{2}\right)^{1 / 2}}$, for $i=1,2$. Combining these two results, we get $\eta_{F}\left(\mu, x_{1}, x_{2}, \mathbf{L}\right)=\sqrt{\eta_{F}^{2}\left(\mu, x_{1}, \mathbf{L}\right)+\eta_{F}^{2}\left(\mu, x_{2}, \mathbf{L}\right)}$.

REMARK 4.5. For T-symmetric/T-skew-symmetric matrix pencils, a relation between the unstructured backward error of a single approximate eigenpair and the structured backward 
error of two approximate eigenpairs of a double-semisimple eigenvalue can be established by using Corollary 4.4 and Corollary 4.3.

REMARK 4.6. From now onwards, we will not invoke the existence theorem separately as we did for the $T$-symmetric/T-skew-symmetric and the unstructured cases in Theorem 3.1 and Theorem 4.1, respectively, because the construction of $\delta A_{0}$ and $\delta A_{1}$ in each case itself guarantees the existence of the required structured matrix pencil.

5. Backward error analysis for Hermitian/skew-Hermitian matrix pencils. This section deals with the backward error analysis of Hermitian and skew-Hermitian matrix pencils. First, we state and prove the main result of this section. Then we establish a relationship between the backward error of a single approximate eigenpair and the backward error of two approximate eigenpairs of a double-semisimple eigenvalue. Before moving to the main result of this section, we now present an important lemma as follows.

LEMMA 5.1. Let $\mathbf{L}$ be a Hermitian/skew-Hermitian matrix pencil of the form (2.1). Let $\lambda=\left(\lambda_{0}, \lambda_{1}\right) \in \mathbb{C}^{2} \backslash\{(0,0)\}$ be a double-semisimple eigenvalue of $\mathbf{L}$ satisfying $\Im\left(\bar{\lambda}_{0} \lambda_{1}\right) \neq 0$, i.e., $\mathbf{L}\left(\lambda_{0}, \lambda_{1}\right) y_{i}=0$, for $i=1,2$, where $y_{1}, y_{2} \in \mathbb{C}^{n}$ are the eigenvectors corresponding to $\lambda$. Then $y_{1}^{H} A_{j} y_{1}=0, y_{2}^{H} A_{j} y_{2}=0$, for $j=0,1$.

Proof. Let $\lambda$ be a double-semisimple eigenvalue of $\mathbf{L}$, i.e., $\left(\lambda_{0} A_{0}+\lambda_{1} A_{1}\right) y_{i}=0$, for $i=1,2$. This gives $y_{i}^{H}\left(\lambda_{0} A_{0}+\lambda_{1} A_{1}\right) y_{i}=0$. Using the fact that $A_{j}=\epsilon A_{j}^{H}$, for $j=0,1$, we get $y_{i}^{H}\left(\lambda_{0} A_{0}+\lambda_{1} A_{1}\right) y_{i}=0$ and $y_{i}^{H}\left(\bar{\lambda}_{0} A_{0}+\bar{\lambda}_{1} A_{1}\right) y_{i}=0$. Solving these two equations along with $\Im\left(\bar{\lambda}_{0} \lambda_{1}\right) \neq 0$, we obtain the desired result.

Throughout this section, $\epsilon=1$ represents the Hermitian case and $\epsilon=-1$ represents the skew-Hermitian case.

REMARK 5.2. For $\epsilon=-1$, we have $\sqrt{\epsilon}=\sqrt{-1}=\iota$, an imaginary number.

REMARK 5.3. Let $\mathbf{L} \in \mathbf{L}\left(\mathbb{C}^{n \times n}\right)$ be a Hermitian/ skew-Hermitian homogeneous matrix pencil of the form (2.1). Suppose that $\left(\lambda, x_{1}\right)$ and $\left(\lambda, x_{2}\right)$ are two approximate eigenpairs of $\mathbf{L}$, with $\lambda=\left(\lambda_{0}, \lambda_{1}\right) \in \mathbb{C}^{2} \backslash\{(0,0)\}$ being a double-semisimple eigenvalue, such that $\Im\left(\bar{\lambda}_{0} \lambda_{1}\right)=0$ and $x_{1}, x_{2} \in \mathbb{C}^{n}$ are orthonormal vectors. Set $k_{i}:=-\mathbf{L}(\lambda) x_{i}$, for $i=1,2$. Then $\lambda_{j} \epsilon \overline{x_{2}^{H} k_{1}}=\bar{\lambda}_{j} x_{1}^{H} k_{2}$, for $j=0,1$, and $\left|\epsilon x_{2}^{H} k_{1}\right|^{2}=\left|x_{1}^{H} k_{2}\right|^{2}$.

Now we state and prove the main result of this section.

THEOREM 5.4. Let $\mathbf{L} \in \mathbf{L}\left(\mathbb{C}^{n \times n}\right)$ be a Hermitian/skew-Hermitian homogeneous matrix pencil of the form (2.1). Suppose that $\left(\lambda, x_{1}\right)$ and $\left(\lambda, x_{2}\right)$ are two approximate eigenpairs of $\mathbf{L}$, where $\lambda=\left(\lambda_{0}, \lambda_{1}\right) \in \mathbb{C}^{2} \backslash\{(0,0)\}$ is a double-semisimple eigenvalue and $x_{1}, x_{2} \in \mathbb{C}^{n}$ are orthonormal vectors. Set $k_{i}:=-\mathbf{L}(\lambda) x_{i}$, for $i=1,2$. Then there exists a Hermitian/ skew-Hermitian matrix pencil $\delta \mathbf{L}$ of the form $\delta \mathbf{L}(\alpha)=\alpha_{0} \delta A_{0}+\alpha_{1} \delta A_{1}$, such that $(\mathbf{L}(\lambda)+$ $\delta \mathbf{L}(\lambda)) x_{i}=0$. The perturbation matrices, for $\Im\left(\bar{\lambda}_{0} \lambda_{1}\right)=0$, are given by

$\delta A_{0}=\sum_{i=1}^{2} \frac{\bar{\lambda}_{0} k_{i} x_{i}^{H}+\epsilon \lambda_{0} x_{i} k_{i}^{H} P_{x_{1}: x_{2}}}{H_{2}^{2}(\lambda)} \quad$ and $\quad \delta A_{1}=\sum_{i=1}^{2} \frac{\bar{\lambda}_{1} k_{i} x_{i}^{H}+\epsilon \lambda_{1} x_{i} k_{i}^{H} P_{x_{1}: x_{2}}}{H_{2}^{2}(\lambda)}$,

and the backward error is given by

$$
\left(\eta_{F}^{\mathbf{S}}\left(\lambda, x_{1}, x_{2}, \mathbf{L}\right)\right)^{2}=\sum_{i=1}^{2}\left(\frac{2\left\|k_{i}\right\|_{2}^{2}-\left|x_{i}^{H} k_{i}\right|^{2}}{H_{2}^{2}(\lambda)}\right)-2 \frac{\left|x_{2}^{H} k_{1}\right|^{2}}{H_{2}^{2}(\lambda)}
$$


The perturbation matrices, for $\Im\left(\bar{\lambda}_{0} \lambda_{1}\right) \neq 0$, are given by

$$
\begin{aligned}
\delta A_{0}=\sum_{i=1}^{2} & \left(-x_{i} x_{i}^{H} A_{0} x_{i} x_{i}^{H}+\bar{\lambda}_{0} \frac{P_{x_{1}: x_{2}} k_{i} x_{i}^{H}}{H_{2}^{2}(\lambda)}+\epsilon \lambda_{0} \frac{x_{i} k_{i}^{H} P_{x_{1}: x_{2}}}{H_{2}^{2}(\lambda)}\right) \\
& +\frac{x_{2}\left(\bar{\lambda}_{1} x_{2}^{H} k_{1}-\epsilon \lambda_{1} \overline{x_{1}^{H} k_{2}}\right) x_{1}^{H}}{\lambda_{0} \bar{\lambda}_{1}-\bar{\lambda}_{0} \lambda_{1}}+\frac{x_{1}\left(\epsilon \lambda_{1} \overline{x_{2}^{H} k_{1}}-\bar{\lambda}_{1} x_{1}^{H} k_{2}\right) x_{2}^{H}}{\bar{\lambda}_{0} \lambda_{1}-\lambda_{0} \bar{\lambda}_{1}}, \\
\delta A_{1}= & \sum_{i=1}^{2}\left(-x_{i} x_{i}^{H} A_{1} x_{i} x_{i}^{H}+\bar{\lambda}_{1} \frac{P_{x_{1}: x_{2}} k_{i} x_{i}^{H}}{H_{2}^{2}(\lambda)}+\epsilon \lambda_{1} \frac{x_{i} k_{i}^{H} P_{x_{1}: x_{2}}}{H_{2}^{2}(\lambda)}\right) \\
& +\frac{x_{2}\left(\epsilon \lambda_{0} \overline{x_{1}^{H} k_{2}}-\bar{\lambda}_{0} x_{2}^{H} k_{1}\right) x_{1}^{H}}{\lambda_{0} \bar{\lambda}_{1}-\bar{\lambda}_{0} \lambda_{1}}+\frac{x_{1}\left(\bar{\lambda}_{0} x_{1}^{H} k_{2}-\epsilon \lambda_{0} \overline{x_{2}^{H} k_{1}}\right) x_{2}^{H}}{\bar{\lambda}_{0} \lambda_{1}-\lambda_{0} \bar{\lambda}_{1}} .
\end{aligned}
$$

In this case, the backward error is given by

$$
\begin{aligned}
& \left(\eta_{F}^{\mathbf{S}}\left(\lambda, x_{1}, x_{2}, \mathbf{L}\right)\right)^{2}=\sum_{i=1}^{2} \sum_{j=0}^{1}\left(\left|x_{i}^{H} A_{j} x_{i}\right|^{2}+2 \frac{\left\|k_{i}\right\|_{2}^{2}-\left|x_{i}^{H} k_{i}\right|^{2}}{H_{2}^{2}(\lambda)}\right) \\
& \quad-\epsilon \frac{\Re\left(\left(\bar{\lambda}_{0}^{2}+\bar{\lambda}_{1}^{2}\right)\left(x_{1}^{H} k_{2}\right)\left(x_{2}^{H} k_{1}\right)\right)}{\left|\Im\left(\bar{\lambda}_{0} \lambda_{1}\right)\right|^{2}}+\frac{\left|x_{2}^{H} k_{1}\right|^{2}+\left|x_{1}^{H} k_{2}\right|^{2}}{2\left|\Im\left(\bar{\lambda}_{0} \lambda_{1}\right)\right|^{2} H_{2}^{2}(\lambda)}\left(H_{2}^{4}(\lambda)-4\left|\Im\left(\bar{\lambda}_{0} \lambda_{1}\right)\right|^{2}\right) .
\end{aligned}
$$

Proof. To construct $\delta A_{j}$, for $j=0,1$, such that $\delta A_{j}=\epsilon \delta A_{j}^{H}$, we consider

$$
\widetilde{\delta A_{j}}=U^{H} \delta A_{j} U={ }_{n-2}^{2}\left[\begin{array}{c|c}
\widehat{\delta A}_{j} & \epsilon \delta B_{j}{ }^{H} \\
\hline \delta B_{j} & \delta D_{j}
\end{array}\right],
$$

where $\widehat{\delta A_{j}}=\left[\begin{array}{cc}\sqrt{\epsilon} \delta a_{j, 11} & \delta a_{j, 12} \\ \epsilon \overline{\delta a_{j, 12}} & \sqrt{\epsilon} \delta a_{j, 22}\end{array}\right]$ with $\delta a_{j, t t} \in \mathbb{R}$, for $t=1,2, \delta B_{j}=\left[\begin{array}{ll}b_{j 1} & b_{j 2}\end{array}\right]$, $\delta D_{j}=\epsilon \delta D_{j}{ }^{H}$, for $j=0,1$, and $U \in \mathbb{C}^{n \times n}$ being a unitary matrix such that $U=\left[\begin{array}{ll}V_{1} & V_{2}\end{array}\right]$ with $V_{1}=\left[\begin{array}{ll}x_{1} & x_{2}\end{array}\right] \in \mathbb{C}^{n \times 2}$. Since we need to construct $\delta \mathbf{L}$ such that $(\mathbf{L}(\lambda)+\delta \mathbf{L}(\lambda)) x_{i}=0$, we get $k_{i}=\delta \mathbf{L}(\lambda) x_{i}$, for $i=1,2$. From $\widetilde{\delta} \mathbf{L}(\lambda)=U^{H} \delta \mathbf{L}(\lambda) U$, we have $\widetilde{\delta} \mathbf{L}(\lambda) U^{H} x_{i}=$ $U^{H} \delta \mathbf{L}(\lambda) x_{i}=U^{H} k_{i}$. This implies

$$
\lambda_{0}\left[\begin{array}{cc}
\widehat{\delta A_{0}} & \epsilon \delta B_{0}^{H} \\
\delta B_{0} & \delta D_{1}
\end{array}\right]\left[\begin{array}{c}
e_{i} \\
0
\end{array}\right]+\lambda_{1}\left[\begin{array}{cc}
\widehat{\delta A_{1}} & \epsilon \delta B_{1}^{H} \\
\delta B_{1} & \delta D_{1}
\end{array}\right]\left[\begin{array}{c}
e_{i} \\
0
\end{array}\right]=\left[\begin{array}{c}
V_{1}^{H} k_{i} \\
V_{2}^{H} k_{i}
\end{array}\right],
$$

and further simplifications give

$$
\left[\begin{array}{l}
\left(\lambda_{0} \widehat{\delta A_{0}}+\lambda_{1} \widehat{\delta A_{1}}\right) e_{i} \\
\left(\lambda_{0} \delta B_{0}+\lambda_{1} \delta B_{1}\right) e_{i}
\end{array}\right]=\left[\begin{array}{l}
V_{1}^{H} k_{i} \\
V_{2}^{H} k_{i}
\end{array}\right]
$$

where $e_{i} \in \mathbb{C}^{2}$ is a vector having 1 at $i^{t h}$ position and 0 elsewhere. From (5.2), we get the following four equations and one system of equation:

$$
\begin{array}{rlrl}
\sqrt{\epsilon} \lambda_{0} \delta a_{0, i i}+\sqrt{\epsilon} \lambda_{1} \delta a_{1, i i} & =x_{i}^{H} k_{i}, & i=1,2, \\
\lambda_{0} b_{0 i}+\lambda_{1} b_{1 i} & =V_{2}^{H} k_{i}, & i=1,2, \\
{\left[\begin{array}{cc}
\lambda_{0} & \bar{\lambda}_{1} \\
\lambda_{0} & \lambda_{1}
\end{array}\right]\left[\begin{array}{c}
\delta a_{0,12} \\
\delta a_{1,12}
\end{array}\right]} & =\left[\begin{array}{c}
\epsilon x_{2}^{H} k_{1} \\
x_{1}^{H} k_{2}
\end{array}\right] .
\end{array}
$$


The minimum norm solution of (5.4) is given by $b_{0 i}=\frac{\bar{\lambda}_{0}}{H_{2}^{2}(\lambda)} V_{2}^{H} k_{i}$ and $b_{1 i}=\frac{\bar{\lambda}_{1}}{H_{2}^{2}(\lambda)} V_{2}^{H} k_{i}$.

Case 1: If $\Im\left(\bar{\lambda}_{0} \lambda_{1}\right)=0$, then the minimum norm solution of (5.3) is given by

$$
\delta a_{0, i i}=\frac{\overline{\sqrt{\epsilon} \lambda_{0}}}{H_{2}^{2}(\lambda)} x_{i}^{H} k_{i}, \quad \delta a_{1, i i}=\frac{\overline{\sqrt{\epsilon} \lambda_{1}}}{H_{2}^{2}(\lambda)} x_{i}^{H} k_{i} .
$$

Since $A_{j}=\epsilon A_{j}^{H}$, for $j=0,1$, and $\Im\left(\bar{\lambda}_{0} \lambda_{1}\right)=0$, we get that the system (5.5) is consistent by using Remark 5.3. The minimum norm solution of (5.5) is given by

$$
\delta a_{0,12}=\frac{\epsilon \lambda_{0}}{H_{2}^{2}(\lambda)} \overline{x_{2}^{H} k_{1}} \quad \text { and } \quad \delta a_{1,12}=\frac{\epsilon \lambda_{1}}{H_{2}^{2}(\lambda)} \overline{x_{2}^{H} k_{1}} .
$$

Substituting back all these obtained entries in (5.1), we get

$$
\delta A_{j}=U\left[\begin{array}{ccc}
\frac{\bar{\lambda}_{j}}{H_{2}^{2}(\lambda)} x_{1}^{H} k_{1} & \epsilon \frac{\lambda_{j}}{\bar{\lambda}_{2}^{2}(\lambda)} \overline{x_{2}^{H} k_{1}} & \epsilon \frac{\lambda_{j}}{H_{2}^{2}(\lambda)}\left(V_{2}^{H} k_{1}\right)^{H} \\
\frac{\lambda_{2}^{2}}{H_{2}^{2}(\lambda)} x_{2}^{H} k_{1} & \frac{\lambda_{j}}{H_{2}^{2}(\lambda)} x_{2}^{H} k_{2} & \epsilon \frac{\lambda_{j}}{H_{2}^{2}(\lambda)}\left(V_{2}^{H} k_{2}\right)^{H} \\
\frac{\lambda_{j}}{H_{2}^{2}(\lambda)} V_{2}^{H} k_{1} & \frac{\lambda_{j}}{H_{2}^{2}(\lambda)} V_{2}^{H} k_{2} & D_{j}
\end{array}\right] U^{H} .
$$

Further simplifying (5.6) and setting $\delta D_{j}=0$ along with Remark 5.3, we get the desired perturbation matrices $\delta A_{0}, \delta A_{1}$ whose Frobenius norms are minimum.

Next we need to show $(\mathbf{L}(\lambda)+\delta \mathbf{L}(\lambda)) x_{i}=0$, for $i=1,2$. Consider

$$
(\mathbf{L}(\lambda)+\delta \mathbf{L}(\lambda)) x_{i}=\mathbf{L}(\lambda) x_{i}+\delta \mathbf{L}(\lambda) x_{i}=-k_{i}+\lambda_{0} \delta A_{0} x_{i}+\lambda_{1} \delta A_{1} x_{i}=-k_{i}+k_{i}=0 .
$$

Since the Frobenius norms of $\delta A_{0}$ and $\delta A_{1}$ are minimal, it follows that

$$
\left(\eta_{F}^{\mathbf{S}}\left(\lambda, x_{1}, x_{2}, \mathbf{L}\right)\right)^{2}=\left\|\delta A_{0}\right\|_{F}^{2}+\left\|\delta A_{1}\right\|_{F}^{2},
$$

where

$$
\left\|\delta A_{0}\right\|_{F}^{2}+\left\|\delta A_{1}\right\|_{F}^{2}=\sum_{j=0}^{1}\left\|\widehat{\delta A_{j}}\right\|_{F}^{2}+2\left\|\delta B_{j}\right\|_{F}^{2}=\sum_{i=1}^{2} \frac{\left|x_{i}^{H} k_{i}\right|^{2}}{H_{2}^{2}(\lambda)}+2 \frac{\left|x_{2}^{H} k_{1}\right|^{2}}{H_{2}^{2}(\lambda)}+2 \frac{\left\|V_{2}^{H} k_{i}\right\|^{2}}{H_{2}^{2}(\lambda)} .
$$

Since $\left\|V_{2}^{H} k_{i}\right\|_{2}^{2}=\left\|k_{i}\right\|_{2}^{2}-\left|x_{1}^{H} k_{i}\right|^{2}-\left|x_{2}^{H} k_{i}\right|^{2}$ and using Remark 5.3, we get

$$
\left(\eta_{F}^{\mathbf{S}}\left(\lambda, x_{1}, x_{2}, \mathbf{L}\right)\right)^{2}=\sum_{i=1}^{2}\left(\frac{2\left\|k_{i}\right\|_{2}^{2}-\left|x_{i}^{T} k_{i}\right|^{2}}{H_{2}^{2}(\lambda)}\right)-2 \frac{\left|x_{2}^{H} k_{1}\right|^{2}}{H_{2}^{2}(\lambda)} .
$$

Case 2: If $\Im\left(\bar{\lambda}_{0} \lambda_{1}\right) \neq 0$, then using Lemma 5.1, we get $\delta a_{0, i i}=-\overline{\sqrt{\epsilon}} x_{i}^{H} A_{0} x_{i}$, $\delta a_{1, i i}=-\overline{\sqrt{\epsilon}} x_{i}^{H} A_{1} x_{i}$. When $\Im\left(\bar{\lambda}_{0} \lambda_{1}\right) \neq 0$, i.e., $\bar{\lambda}_{0} \lambda_{1}-\lambda_{0} \bar{\lambda}_{1} \neq 0$, the unique solution of the system (5.5) is given by $\delta a_{0,12}=\frac{\epsilon \lambda_{1} \overline{x_{2}^{H} k_{1}}-\bar{\lambda}_{1} x_{1}^{H} k_{2}}{\bar{\lambda}_{0} \lambda_{1}-\lambda_{0} \bar{\lambda}_{1}}$ and $\delta a_{1,12}=\frac{-\epsilon \lambda_{0} \overline{x_{2}^{H} k_{1}}+\bar{\lambda}_{0} x_{1}^{H} k_{2}}{\bar{\lambda}_{0} \lambda_{1}-\lambda_{0} \bar{\lambda}_{1}}$, which is the minimum norm solution. Similar to Case 1, we get the desired perturbed matrices by substituting back the obtained entries in (5.1). In this case

$$
\left(\eta_{F}^{\mathbf{S}}\left(\lambda, x_{1}, x_{2}, \mathbf{L}\right)\right)^{2}=\left\|\delta A_{0}\right\|_{F}^{2}+\left\|\delta A_{1}\right\|_{F}^{2},
$$

where

$$
\begin{aligned}
\left\|\delta A_{0}\right\|_{F}^{2} & +\left\|\delta A_{1}\right\|_{F}^{2}=\sum_{j=0}^{1} \sum_{i=1}^{2}\left|x_{i}^{H} A_{j} x_{i}\right|^{2}+\sum_{i=1}^{2} \frac{2\left\|k_{i}\right\|^{2}-2\left|x_{i}^{H} k_{i}\right|^{2}}{H_{2}^{2}(\lambda)} \\
+ & 2 \frac{\left|\epsilon \lambda_{1} \overline{x_{2}^{H} k_{1}}-\bar{\lambda}_{1} x_{1}^{H} k_{2}\right|^{2}+\left|\lambda_{0} x_{1}^{H} k_{2}-\epsilon \lambda_{0} x_{2}^{H} k_{1}\right|^{2}}{\left|\bar{\lambda}_{0} \lambda_{1}-\lambda_{0} \bar{\lambda}_{1}\right|^{2}}-2 \frac{\left|x_{1}^{H} r_{2}\right|^{2}+\left|x_{2}^{H} r_{1}\right|^{2}}{H_{2}^{2}(\lambda)} .
\end{aligned}
$$


Since

$$
\begin{aligned}
& \frac{\left|\epsilon \lambda_{1} \overline{x_{2}^{H} k_{1}}-\bar{\lambda}_{1} x_{1}^{H} k_{2}\right|^{2}+\left|\lambda_{0} x_{1}^{H} k_{2}-\epsilon \lambda_{0} x_{2}^{H} k_{1}\right|^{2}}{\left|\bar{\lambda}_{0} \lambda_{1}-\lambda_{0} \bar{\lambda}_{1}\right|^{2}} \\
& =\left(H_{2}^{2}(\lambda) \frac{\left|x_{2}^{H} k_{1}\right|^{2}+\left|x_{1}^{H} k_{2}\right|^{2}}{4\left|\Im\left(\overline{\lambda_{0}} \lambda_{1}\right)\right|^{2}}-\epsilon \frac{\Re\left(\left(\bar{\lambda}_{0}^{2}+\bar{\lambda}_{1}^{2}\right)\left(x_{1}^{H} k_{2}\right)\left(x_{2}^{H} k_{1}\right)\right)}{2\left|\Im\left(\overline{\lambda_{0}} \lambda_{1}\right)\right|^{2}}\right),
\end{aligned}
$$

we get

$$
\begin{gathered}
\left(\eta_{F}^{\mathbf{S}}\left(\lambda, x_{1}, x_{2}, \mathbf{L}\right)\right)^{2}=\sum_{i=1}^{2} \sum_{j=0}^{1}\left(\left|x_{i}^{H} A_{j} x_{i}\right|^{2}+2 \frac{\left\|k_{i}\right\|_{2}^{2}-\left|x_{i}^{H} k_{i}\right|^{2}}{H_{2}^{2}(\lambda)}\right) \\
-\epsilon \frac{\Re\left(\left(\bar{\lambda}_{0}^{2}+\bar{\lambda}_{1}^{2}\right)\left(x_{1}^{H} k_{2}\right)\left(x_{2}^{H} k_{1}\right)\right)}{\left|\Im\left(\bar{\lambda}_{0} \lambda_{1}\right)\right|^{2}}+\frac{\left|x_{2}^{H} k_{1}\right|^{2}+\left|x_{1}^{H} k_{2}\right|^{2}}{2\left|\Im\left(\bar{\lambda}_{0} \lambda_{1}\right)\right|^{2} H_{2}^{2}(\lambda)}\left(H_{2}^{4}(\lambda)-4\left|\Im\left(\bar{\lambda}_{0} \lambda_{1}\right)\right|^{2}\right) .
\end{gathered}
$$

COROLlary 5.5. Let $\mathbf{L}$ be a non-homogeneous Hermitian/skew-Hermitian matrix pencil of the form (2.1). Let $\left(\mu, x_{1}\right)$ and $\left(\mu, x_{2}\right)$ be two approximate eigenpairs, where $\mu \in \mathbb{R}$ is a double-semisimple eigenvalue and $x_{1}, x_{2} \in \mathbb{C}^{n}$ are orthonormal vectors. Set $k_{i}:=-\mathbf{L}(\mu) x_{i}$. Then the following inequality holds:

$$
\left(\eta_{F}^{\mathbf{S}}\left(\mu, x_{1}, x_{2}, \mathbf{L}\right)\right) \leq \sqrt{\eta_{F}^{\mathbf{S}}\left(\mu, x_{1}, \mathbf{L}\right)^{2}+\eta_{F}^{\mathbf{S}}\left(\mu, x_{2}, \mathbf{L}\right)^{2}} .
$$

Proof. Substituting $\lambda_{0}=1, \lambda_{1}=\mu$ in Theorem 5.4 and using [1, Theorem 3.6], we get the desired backward error relation.

REMARK 5.6. Let $\left(\mu, x_{1}\right)$ and $\left(\mu, x_{2}\right)$ be two approximate eigenpairs of a non-homogeneous Hermitian/skew-Hermitian matrix pencil $\mathbf{L}$, where $\mu \in \mathbb{C}$ is a double-semisimple eigenvalue and $x_{1}, x_{2} \in \mathbb{C}^{n}$ are orthonormal eigenvectors. Then similar to Corollary 3.7, substituting $\lambda_{0}=1, \lambda_{1}=\mu$ in Theorem 5.4, and using [1, Theorem 3.6], we get

$$
\begin{aligned}
\eta_{F}^{\mathbf{S}}\left(\mu, x_{1}, x_{2}, \mathbf{L}\right) & =\sqrt{\left(\eta_{F}^{\mathbf{S}}\left(\mu, x_{1}, \mathbf{L}\right)\right)^{2}+\left(\eta_{F}^{\mathbf{S}}\left(\mu, x_{2}, \mathbf{L}\right)\right)^{2}} \\
& =\sqrt{2} \sqrt{\left(\eta_{F}\left(\mu, x_{1}, \mathbf{L}\right)\right)^{2}+\left(\eta_{F}\left(\mu, x_{2}, \mathbf{L}\right)\right)^{2}}
\end{aligned}
$$

when $\mu^{2}=-1$. Further, using Corollary 4.4 in the above relation, we get

$$
\eta_{F}^{\mathbf{S}}\left(\mu, x_{1}, x_{2}, \mathbf{L}\right)=\sqrt{2}\left(\eta_{F}\left(\mu, x_{1}, x_{2}, \mathbf{L}\right)\right),
$$

when $\mu^{2}=-1$. Note that for $\mu^{2}=-1$, we have $H_{2}^{4}(\lambda)-4\left|\Im\left(\bar{\lambda}_{0} \lambda_{1}\right)\right|^{2}=0$.

Similar to Hermitian/skew-Hermitian matrix pencils, next we present the backward error analysis for $H$-even/H-odd matrix pencils.

6. Backward error analysis for $\boldsymbol{H}$-even/H-odd matrix pencils. In this section, we discuss the backward error analysis for $H$-even and $H$-odd matrix pencils. We start this section with the following important lemma.

Lemma 6.1. Let $\mathbf{L}$ be an H-even/H-odd matrix pencil of the form (2.1). Let $\lambda=$ $\left(\lambda_{0}, \lambda_{1}\right) \in \mathbb{C}^{2} \backslash\{(0,0)\}$ be a double-semisimple eigenvalue of $\mathbf{L}$ satisfying $\Re\left(\bar{\lambda}_{0} \lambda_{1}\right) \neq 0$, i.e., $\mathbf{L}\left(\lambda_{0}, \lambda_{1}\right) y_{i}=0$, for $i=1,2$, where $y_{1}, y_{2} \in \mathbb{C}^{n}$ are the eigenvectors corresponding to $\lambda$. Then $y_{1}^{H} A_{j} y_{1}=0, y_{2}^{H} A_{j} y_{2}=0$, for $j=0,1$.

Proof. The proof follows similar to Lemma 5.1 by using the fact that $A_{0}=\epsilon A_{0}^{H}$ and $A_{1}=-\epsilon A_{1}^{H}$.

Throughout this section, $\epsilon=1$ represents the $H$-even case and $\epsilon=-1$ represents the $H$-odd case. 
REMARK 6.2. Let $\mathbf{L} \in \mathbf{L}\left(\mathbb{C}^{n \times n}\right)$ be an H-even/ H-odd homogeneous matrix pencil of the form (2.1). Suppose that $\left(\lambda, x_{1}\right)$ and $\left(\lambda, x_{2}\right)$ are two approximate eigenpairs of $\mathbf{L}$, where $\lambda=\left(\lambda_{0}, \lambda_{1}\right) \in \mathbb{C}^{2} \backslash\{(0,0)\}$ is a double-semisimple eigenvalue such that $\Re\left(\bar{\lambda}_{0} \lambda_{1}\right)=0$ and $x_{1}, x_{2} \in \mathbb{C}^{n}$ are orthonormal vectors. Let $k_{i}:=-\mathbf{L}(\lambda) x_{i}$, for $i=1,2$. Then $\lambda_{0} \epsilon \overline{x_{2}^{H} k_{1}}=$ $\bar{\lambda}_{0} x_{1}^{H} k_{2}, \lambda_{1} \epsilon \overline{x_{2}^{H} k_{1}}=-\bar{\lambda}_{1} x_{1}^{H} k_{2}$, and $\left|\epsilon x_{2}^{H} k_{1}\right|^{2}=\left|x_{1}^{H} k_{2}\right|^{2}$.

Now we state and prove the main result of this section.

THEOREM 6.3. Let $\mathbf{L} \in \mathbf{L}\left(\mathbb{C}^{n \times n}\right)$ be a H-even/H-odd matrix pencil of the form (2.1). Let $\left(\lambda, x_{1}\right)$ and $\left(\lambda, x_{2}\right)$ be two approximate eigenpairs of $\mathbf{L}$, where $x_{1}, x_{2} \in \mathbb{C}^{n}$ are orthonormal vectors and $\lambda=\left(\lambda_{0}, \lambda_{1}\right) \in \mathbb{C}^{2} \backslash\{(0,0)\}$ is a double-semisimple eigenvalue. Set $k_{i}=$ $-\mathbf{L}(\lambda) x_{i}$, for $i=1,2$. Then there exists an $H$-even/H-odd matrix pencil $\delta \mathbf{L}$ of the form $\delta \mathbf{L}(\alpha)=\alpha_{0} \delta A_{0}+\alpha_{1} \delta A_{1}$, such that $(\mathbf{L}(\lambda)+\delta \mathbf{L}(\lambda)) x_{i}=0$. The perturbation matrices, for $\Re\left(\bar{\lambda}_{0} \lambda_{1}\right)=0$, are given by

$\delta A_{0}=\sum_{i=1}^{2} \frac{\bar{\lambda}_{0} k_{i} x_{i}^{H}+\epsilon \lambda_{0} x_{i} k_{i}^{H} P_{x_{1}: x_{2}}}{H_{2}^{2}(\lambda)} \quad$ and $\quad \delta A_{1}=\sum_{i=1}^{2} \frac{\bar{\lambda}_{1} k_{i} x_{i}^{H}-\epsilon \lambda_{1} x_{i} k_{i}^{H} P_{x_{1}: x_{2}}}{H_{2}^{2}(\lambda)}$.

In this case, the backward error is given by

$$
\left(\eta_{F}^{\mathbf{S}}\left(\lambda, x_{1}, x_{2}, \mathbf{L}\right)\right)^{2}=\sum_{i=1}^{2}\left(\frac{2\left\|k_{i}\right\|_{2}^{2}-\left|x_{i}^{T} k_{i}\right|^{2}}{H_{2}^{2}(\lambda)}\right)-2 \frac{\left|x_{1}^{H} k_{2}\right|^{2}}{H_{2}^{2}(\lambda)} .
$$

The perturbation matrices, for $\Re\left(\bar{\lambda}_{0} \lambda_{1}\right) \neq 0$, are given by

$$
\begin{aligned}
\delta A_{0}=\sum_{i=1}^{2} & \left(-x_{i} x_{i}^{H} A_{0} x_{i} x_{i}^{H}+\bar{\lambda}_{0} \frac{P_{x_{1}: x_{2}} k_{i} x_{i}^{H}}{H_{2}^{2}(\lambda)}+\epsilon \lambda_{0} \frac{x_{i} k_{i}^{H} P_{x_{1}: x_{2}}}{H_{2}^{2}(\lambda)}\right) \\
& +\frac{x_{2}\left(\bar{\lambda}_{1} x_{2}^{H} k_{1}+\epsilon \lambda_{1} \overline{x_{1}^{H} k_{2}}\right) x_{1}^{H}}{\bar{\lambda}_{0} \lambda_{1}+\lambda_{0} \bar{\lambda}_{1}}+\frac{x_{1}\left(\epsilon \lambda_{1} \overline{x_{2}^{H} k_{1}}+\bar{\lambda}_{1} x_{1}^{H} k_{2}\right) x_{2}^{H}}{\bar{\lambda}_{0} \lambda_{1}+\lambda_{0} \bar{\lambda}_{1}}, \\
\delta A_{1}= & \sum_{i=1}^{2}\left(-x_{i} x_{i}^{H} A_{1} x_{i} x_{i}^{H}+\bar{\lambda}_{1} \frac{P_{x_{1}: x_{2}} k_{i} x_{i}^{H}}{H_{2}^{2}(\lambda)}-\epsilon \lambda_{1} \frac{x_{i} k_{i}^{H} P_{x_{1}: x_{2}}}{H_{2}^{2}(\lambda)}\right) \\
& +\frac{x_{2}\left(\bar{\lambda}_{0} x_{2}^{H} k_{1}-\epsilon \lambda_{0} \overline{x_{1}^{H} k_{2}}\right) x_{1}^{H}}{\bar{\lambda}_{0} \lambda_{1}+\lambda_{0} \bar{\lambda}_{1}}+\frac{x_{1}\left(\bar{\lambda}_{0} x_{1}^{H} k_{2}-\epsilon \lambda_{0} \overline{x_{2}^{H} k_{1}}\right) x_{2}^{H}}{\bar{\lambda}_{0} \lambda_{1}+\lambda_{0} \bar{\lambda}_{1}} .
\end{aligned}
$$

In this case, the backward error is given by

$$
\begin{aligned}
& \left(\eta_{F}^{\mathbf{S}}\left(\lambda, x_{1}, x_{2}, \mathbf{L}\right)\right)^{2}=\sum_{i=1}^{2} \sum_{j=0}^{1}\left(\left|x_{i}^{H} A_{j} x_{i}\right|^{2}+\frac{2\left\|k_{i}\right\|_{2}^{2}-2\left|x_{i}^{H} k_{i}\right|^{2}}{H_{2}^{2}(\lambda)}\right) \\
& \quad+\epsilon \frac{\Re\left(\left(\bar{\lambda}_{1}^{2}-\bar{\lambda}_{0}^{2}\right)\left(x_{1}^{H} k_{2}\right)\left(x_{2}^{H} k_{1}\right)\right)}{\left|\Re\left(\bar{\lambda}_{0} \lambda_{1}\right)\right|^{2}}+\frac{\left|x_{2}^{H} k_{1}\right|^{2}+\left|x_{1}^{H} k_{2}\right|^{2}}{2\left|\Re\left(\bar{\lambda}_{0} \lambda_{1}\right)\right|^{2} H_{2}^{2}(\lambda)}\left(H_{2}^{4}(\lambda)-4\left|\Re\left(\bar{\lambda}_{0} \lambda_{1}\right)\right|^{2}\right) .
\end{aligned}
$$

Proof. To construct $\delta A_{j}$, for $j=0,1$, such that $\delta A_{0}=\epsilon \delta A_{0}^{H}$ and $\delta A_{1}=-\epsilon \delta A_{1}^{H}$, we consider

$$
\begin{gathered}
\widetilde{\delta A_{0}}=U^{H} \delta A_{0} U=\underset{n-2}{2}\left[\begin{array}{c|c}
\widehat{\delta A}_{0} & \epsilon \delta B_{0}{ }^{H} \\
\hline \delta B_{0} & \delta D_{0}
\end{array}\right], \\
\widetilde{\delta A_{1}}=U^{H} \delta A_{1} U=\underset{n-2}{2}\left[\begin{array}{c|c}
\widehat{\delta A}_{1} & -\epsilon \delta B_{1}{ }^{H} \\
\hline \delta B_{1} & \delta D_{1}
\end{array}\right],
\end{gathered}
$$


where

$$
\begin{aligned}
\widehat{\delta A_{0}} & =\left[\begin{array}{cc}
\sqrt{\epsilon} \delta a_{0,11} & \delta a_{0,12} \\
\epsilon \overline{\delta a_{0,12}} & \sqrt{\epsilon} \delta a_{0,22}
\end{array}\right], \quad \widehat{\delta A_{1}}=\left[\begin{array}{cc}
\sqrt{-\epsilon} \delta a_{1,11} & \delta a_{1,12} \\
-\epsilon \overline{\delta a_{1,12}} & \sqrt{-\epsilon} \delta a_{1,22}
\end{array}\right], \\
\delta D_{0} & =\epsilon \delta D_{0}{ }^{H}, \quad \delta D_{1}=-\epsilon \delta D_{1}{ }^{H}, \quad \delta B_{j}=\left[\begin{array}{ll}
b_{j 1} & b_{j 2}
\end{array}\right],
\end{aligned}
$$

for $j=0,1$, and $U \in \mathbb{C}^{n \times n}$ is a unitary matrix such that $U=\left[\begin{array}{ll}V_{1} & V_{2}\end{array}\right]$ with $V_{1}=\left[\begin{array}{ll}x_{1} & x_{2}\end{array}\right]$ $\in \mathbb{C}^{n \times 2}$. Similar to Theorem 5.4, we get the following equations

$$
\begin{aligned}
\sqrt{\epsilon} \lambda_{0} \delta a_{0, i i}+\sqrt{-\epsilon} \lambda_{1} \delta a_{1, i i} & =x_{i}^{H} k_{i}, & i=1,2, \\
\lambda_{0} b_{0 i}+\lambda_{1} b_{1 i} & =V_{2}^{H} k_{i}, & i=1,2, \\
{\left[\begin{array}{cc}
\bar{\lambda}_{0} & -\bar{\lambda}_{1} \\
\lambda_{0} & \lambda_{1}
\end{array}\right]\left[\begin{array}{l}
\delta a_{0,12} \\
\delta a_{1,12}
\end{array}\right] } & =\left[\begin{array}{c}
\epsilon x_{2}^{H} k_{1} \\
x_{1}^{H} k_{2}
\end{array}\right] . &
\end{aligned}
$$

The minimum norm solution of (6.4) is given by $b_{0 i}=\frac{\bar{\lambda}_{0}}{H_{2}^{2}(\lambda)} V_{2}^{H} k_{i}, b_{1 i}=\frac{\bar{\lambda}_{1}}{H_{2}^{2}(\lambda)} V_{2}^{H} k_{i}$. Next, we have the following two cases.

Case 1: If $\Re\left(\bar{\lambda}_{0} \lambda_{1}\right)=0$, then the minimum norm solution of (6.3) is given by $\delta a_{0, i i}=$ $\frac{\overline{\sqrt{\epsilon} \lambda_{0}}}{H_{2}^{2}(\lambda)} x_{i}^{H} k_{i}, \delta a_{1, i i}=\frac{\overline{\sqrt{-\epsilon} \lambda_{1}}}{H_{2}^{2}(\lambda)} x_{i}^{H} k_{i}$. Since $A_{0}=\epsilon A_{0}^{H}, A_{0}=-\epsilon A_{0}^{H}$ and $\Re\left(\bar{\lambda}_{0} \lambda_{1}\right)=0$, we get that the system (6.5) is consistent by using Remark 6.2. The minimum norm solution of (6.5) is given by $\delta a_{0,12}=\frac{\epsilon \lambda_{0}}{H_{2}^{2}(\lambda)} \overline{x_{2}^{H} k_{1}}$ and $\delta a_{1,12}=\frac{-\epsilon \lambda_{1}}{H_{2}^{2}(\lambda)} \overline{x_{2}^{H} k_{1}}$. Substituting these obtained values in (6.1) and (6.2), we get the desired perturbed matrices and backward error.

Case 2: If $\Re\left(\bar{\lambda}_{0} \lambda_{1}\right) \neq 0$, then using Lemma 6.1, we get $\delta a_{0, i i}=-\overline{\sqrt{\epsilon}} x_{i}^{H} A_{0} x_{i}$, and $\delta a_{1, i i}=-\overline{\sqrt{-\epsilon}} x_{i}^{H} A_{1} x_{i}$. Since $\Re\left(\bar{\lambda}_{0} \lambda_{1}\right) \neq 0$, i.e., $\bar{\lambda}_{0} \lambda_{1}+\lambda_{0} \bar{\lambda}_{1} \neq 0$, the unique solution of the system (6.5) is given by

$$
\delta a_{0,12}=\frac{\epsilon \lambda_{1} \overline{x_{2}^{H} k_{1}}+\bar{\lambda}_{1} x_{1}^{H} k_{2}}{\bar{\lambda}_{0} \lambda_{1}+\lambda_{0} \bar{\lambda}_{1}}, \quad \delta a_{1,12}=\frac{-\epsilon \lambda_{0} \overline{x_{2}^{H} k_{1}}+\bar{\lambda}_{0} x_{1}^{H} k_{2}}{\bar{\lambda}_{0} \lambda_{1}+\lambda_{0} \bar{\lambda}_{1}},
$$

which is the minimum norm solution. Using these obtained values, we can get the desired $\delta A_{0}, \delta A_{1}$ and the backward error in this case.

REMARK 6.4. Suppose $\left(\mu, x_{1}\right)$ and $\left(\mu, x_{2}\right)$ are two approximate eigenpairs of a nonhomogeneous $H$-even/H-odd matrix pencil $\mathbf{L}$ such that $\mu \in \mathbb{C}$ is a double-semisimple eigenvalue and $x_{1}, x_{2} \in \mathbb{C}^{n}$ are orthonormal vectors. Then similar to Corollary 3.7 , substituting $\lambda_{0}=1, \lambda_{1}=\mu$ in Theorem 6.3, and using [1, Theorem 3.7], we get

$$
\begin{aligned}
\left(\eta_{F}^{\mathbf{S}}\left(\mu, x_{1}, x_{2}, \mathbf{L}\right)\right)^{2} & =\left(\eta_{F}^{\mathbf{S}}\left(\mu, x_{1}, \mathbf{L}\right)\right)^{2}+\left(\eta_{F}^{\mathbf{S}}\left(\mu, x_{2}, \mathbf{L}\right)\right)^{2} \\
& =2\left(\left(\eta_{F}\left(\mu, x_{1}, \mathbf{L}\right)\right)^{2}+\left(\eta_{F}\left(\mu, x_{2}, \mathbf{L}\right)\right)^{2}\right),
\end{aligned}
$$

for $\mu^{2}=1$. Further, using Corollary 4.4 in this obtained relation, we get

$$
\eta_{F}^{\mathbf{S}}\left(\mu, x_{1}, x_{2}, \mathbf{L}\right)=\sqrt{2}\left(\eta_{F}\left(\mu, x_{1}, x_{2}, \mathbf{L}\right)\right),
$$

when $\mu^{2}=1$. Note that for $\mu^{2}=1$, we have $H_{2}^{4}(\lambda)-4\left|\Re\left(\bar{\lambda}_{0} \lambda_{1}\right)\right|^{2}=0$.

In the following section, we discuss the backward error analysis for $T$-even $/ T$-odd matrix pencils.

7. Backward error analysis for $T$-even/T-odd matrix pencils. In this section, we state and prove the structured backward error theorem for $T$-even $/ T$-odd matrix pencils. The derivation of the theorem is similar to the previous section. Hence we discuss only those steps which are unique for this section. We start this section with two important lemmas. 
Lemma 7.1. Suppose that $\lambda=\left(\lambda_{0}, \lambda_{1}\right) \in \mathbb{C}^{2}$ with $\lambda_{0} \neq 0, \lambda_{1} \neq 0$, and let $\epsilon=1,-1$. Then the following equality holds:

$$
\frac{1}{G_{\epsilon}^{2}(\lambda)}-\frac{1}{H^{2}(\lambda)}=\frac{\epsilon\left(\left|\lambda_{1}\right|^{2}-\left|\lambda_{0}\right|^{2}\right)}{G_{\epsilon}^{2}(\lambda) H^{2}(\lambda)}
$$

where $G_{\epsilon}(\lambda)=\sqrt{\frac{\left|\lambda_{0}\right|^{2}(1+\epsilon)+\left|\lambda_{1}\right|^{2}(1-\epsilon)}{2}}$ and $H_{2}(\lambda)=\left(\left|\lambda_{0}\right|^{2}+\left|\lambda_{1}\right|^{2}\right)^{1 / 2}$.

Proof. The proof follows from the definitions of $G_{\epsilon}(\lambda)$ and $H_{2}(\lambda)$.

Throughout this section, $\epsilon=1$ and $\epsilon=-1$ represent $T$-even and $T$-odd cases, respectively.

LemMa 7.2. Let $\mathbf{L} \in \mathbf{L}\left(\mathbb{C}^{n \times n}\right)$ be a $T$-even/T-odd homogeneous matrix pencil of the form (2.1). Suppose that $\left(\lambda, x_{1}\right)$ and $\left(\lambda, x_{2}\right)$ are two approximate eigenpairs of $\mathbf{L}$, where $\lambda=\left(\lambda_{0}, \lambda_{1}\right) \in \mathbb{C}^{2} \backslash\{(0,0)\}$ is a double-semisimple eigenvalue and $x_{1}, x_{2} \in \mathbb{C}^{n}$ are orthonormal vectors. Set $k_{i}=-\mathbf{L}(\lambda) x_{i}$, for $i=1,2$. Then the following equality holds for $t=1,2$,

$$
\left(\sum_{i=1}^{2} \sum_{j=1, j \neq i}^{2} \bar{x}_{j} x_{j}^{T} k_{i} x_{i}^{H}\right) x_{t}=\sum_{j=1, j \neq t}^{2} \overline{x_{j}} x_{j}^{T} k_{t} .
$$

Proof. The proof follows, with some simple calculations, from the fact that $x_{1}$ and $x_{2}$ are orthonormal vectors.

REMARK 7.3. For $\lambda_{0}=0$, we have $x_{2}^{T} k_{1}=-\epsilon x_{1}^{T} k_{2}$, and for $\lambda_{1}=0$ we have $x_{2}^{T} k_{1}=\epsilon x_{1}^{T} k_{2}$.

REMARK 7.4. We have $(1+\epsilon)^{2} / 4=(1+\epsilon) / 2$, for $\epsilon=1,-1$.

Now we present the main theorem of this section.

THEOREM 7.5. Let $\mathbf{L} \in \mathbf{L}\left(\mathbb{C}^{n \times n}\right)$ be a T-even/T-odd matrix pencil of the form (2.1). Let $\left(\lambda, x_{1}\right)$ and $\left(\lambda, x_{2}\right)$ be two approximate eigenpairs of $\mathbf{L}$, where $x_{1}, x_{2} \in \mathbb{C}^{n}$ are orthonormal vectors and $\lambda=\left(\lambda_{0}, \lambda_{1}\right) \in \mathbb{C}^{2} \backslash\{(0,0)\}$ is a double-semisimple eigenvalue. Set $k_{i}:=$ $-\mathbf{L}(\lambda) x_{i}$, for $i=1,2$. Then there exists a T-even/T-odd matrix pencil $\delta \mathbf{L}$ of the form $\delta \mathbf{L}(\alpha)=\alpha_{0} \delta A_{0}+\alpha_{1} \delta A_{1}$, such that $(\mathbf{L}(\lambda)+\delta \mathbf{L}(\lambda)) x_{i}=0$. And we have

Case 1: If $\lambda_{0} \neq 0$ and $\lambda_{1} \neq 0$, then the perturbation matrices are given by

$$
\begin{aligned}
\delta A_{0}= & \sum_{i=1}^{2}\left(\bar{\lambda}_{0} \frac{(1+\epsilon)}{2} \frac{\bar{x}_{i} x_{i}^{T} k_{i} x_{i}^{H}}{G_{\epsilon}^{2}(\lambda)}+\bar{\lambda}_{0} \frac{\bar{P}_{x_{1}: x_{2}} k_{i} x_{i}^{H}+\epsilon \bar{x}_{i} k_{i}^{T} P_{x_{1}: x_{2}}}{H_{2}^{2}(\lambda)}\right) \\
& +\sum_{i=1}^{2} \sum_{j=1, j \neq i}^{2} \frac{\bar{x}_{j} x_{j}^{T} k_{i} x_{i}^{H}+\epsilon \bar{x}_{j} x_{i}^{T} k_{j} x_{i}^{H}}{2 \lambda_{0}}, \\
\delta A_{1}= & \sum_{i=1}^{2}\left(\bar{\lambda}_{1} \frac{(1-\epsilon)}{2} \frac{\bar{x}_{i} x_{i}^{T} k_{i} x_{i}^{H}}{G_{\epsilon}^{2}(\lambda)}+\bar{\lambda}_{1} \frac{\bar{P}_{x_{1}: x_{2}} k_{i} x_{i}^{H}-\epsilon \bar{x}_{i} k_{i}^{T} P_{x_{1}: x_{2}}}{H_{2}^{2}(\lambda)}\right) \\
& +\sum_{i=1}^{2} \sum_{j=1, j \neq i}^{2} \frac{\bar{x}_{j} x_{j}^{T} k_{i} x_{i}^{H}-\epsilon \bar{x}_{j} x_{i}^{T} k_{j} x_{i}^{H}}{2 \lambda_{1}},
\end{aligned}
$$

where $G_{\epsilon}(\lambda)=\sqrt{\frac{\left|\lambda_{0}\right|^{2}(1+\epsilon)+\left|\lambda_{1}\right|^{2}(1-\epsilon)}{2}}$. In this case, the backward error is given by

$$
\left(\eta_{F}^{\mathbf{S}}\left(\lambda, x_{1}, x_{2}, \mathbf{L}\right)\right)^{2}=\sum_{i=1}^{2}\left(\frac{2\left\|k_{i}\right\|_{2}^{2}}{H_{2}^{2}(\lambda)}+\frac{\epsilon\left(\left|\lambda_{1}\right|^{2}-\left|\lambda_{0}\right|^{2}\right)\left|x_{i}^{T} k_{i}\right|^{2}}{G_{\epsilon}^{2}(\lambda) H_{2}^{2}(\lambda)}\right)
$$




$$
+\frac{\left|x_{2}^{T} k_{1}\right|^{2}+\left|x_{1}^{T} k_{2}\right|^{2}}{2 H_{2}^{2}(\lambda)}\left(\frac{\left|\lambda_{0}\right|}{\left|\lambda_{1}\right|}-\frac{\left|\lambda_{1}\right|}{\left|\lambda_{0}\right|}\right)^{2}-\left(\frac{\epsilon}{\left|\lambda_{1}\right|^{2}}-\frac{\epsilon}{\left|\lambda_{0}\right|^{2}}\right) \Re\left(\left(x_{1}^{T} k_{2}\right)\left(\overline{x_{2}^{T} k_{1}}\right)\right) .
$$

Case 2: If either $\lambda_{0}=0$ or $\lambda_{1}=0$, then we have the following two cases:

(i) If $\lambda_{0}=0$ and $\lambda_{1} \neq 0$, then the perturbation matrices are given by $\delta A_{0}=0$ and

$$
\begin{aligned}
\delta A_{1}= & \sum_{i=1}^{2}\left(-\frac{(1-\epsilon)}{2} \bar{x}_{i} x_{i}^{T} A_{1} x_{i} x_{i}^{H}-\bar{P}_{x_{1}: x_{2}} A_{1} x_{i} x_{i}^{H}+\bar{x}_{i} x_{i}^{T} A_{1} P_{x_{1}: x_{2}}\right) \\
& +\frac{x_{2}^{T} k_{1}}{\lambda_{1}}\left(\bar{x}_{2} x_{1}^{H}-\epsilon \bar{x}_{1} x_{2}^{H}\right) .
\end{aligned}
$$

In this case the backward error is given by

$$
\left(\eta_{F}^{\mathbf{S}}\left(\lambda, x_{1}, x_{2}, \mathbf{L}\right)\right)^{2}=\sum_{i=1}^{2} 2\left\|A_{1} x_{i}\right\|_{2}^{2}-\frac{(1-\epsilon)}{2}\left|x_{i}^{T} A_{0} x_{i}\right|^{2}-2\left|x_{1}^{T} A_{1} x_{2}\right|^{2} .
$$

(ii) If $\lambda_{0} \neq 0$ and $\lambda_{1}=0$, then the perturbation matrices are given by $\delta A_{1}=0$ and

$$
\begin{aligned}
\delta A_{0}= & \sum_{i=1}^{2}\left(-\frac{(1+\epsilon)}{2} \bar{x}_{i} x_{i}^{T} A_{0} x_{i} x_{i}^{H}-\bar{P}_{x_{1}: x_{2}} A_{0} x_{i} x_{i}^{H}-\bar{x}_{i} x_{i}^{T} A_{0} P_{x_{1}: x_{2}}\right) \\
& +\frac{x_{2}^{T} k_{1}}{\lambda_{0}}\left(\bar{x}_{2} x_{1}^{H}+\epsilon \bar{x}_{1} x_{2}^{H}\right) .
\end{aligned}
$$

In this case, the backward error is given by

$$
\left(\eta_{F}^{\mathbf{S}}\left(\lambda, x_{1}, x_{2}, \mathbf{L}\right)\right)^{2}=\sum_{i=1}^{2} 2\left\|A_{0} x_{i}\right\|_{2}^{2}-\frac{(1+\epsilon)}{2}\left|x_{i}^{T} A_{0} x_{i}\right|^{2}-2\left|x_{1}^{T} A_{0} x_{2}\right|^{2} .
$$

Proof. To construct $\delta A_{j}$, for $j=0,1$, such that $\delta A_{0}=\epsilon \delta A_{0}^{T}$ and $\delta A_{1}=-\epsilon \delta A_{1}^{T}$, we consider

$$
\begin{aligned}
& \widetilde{\delta A_{0}}=U^{T} \delta A_{0} U=\underset{n-2}{2}\left[\begin{array}{c|c}
\stackrel{2}{\delta A_{0}} & \epsilon \delta B_{0}^{T} \\
\hline \delta B_{0} & \delta D_{0}
\end{array}\right], \\
& \widetilde{\delta A_{1}}=U^{T} \delta A_{1} U=\underset{n-2}{2}\left[\begin{array}{c|c}
\widehat{\delta A}_{1} & -\epsilon \delta B_{1}^{T} \\
\hline \delta B_{1} & \delta D_{1}
\end{array}\right],
\end{aligned}
$$

where

$$
\begin{array}{rlr}
\widehat{\delta A_{0}} & =\left[\begin{array}{cc}
\frac{(1+\epsilon)}{2} \delta a_{0,11} & \epsilon \delta a_{0,12} \\
\delta a_{0,12} & \frac{(1+\epsilon)}{2} \delta a_{0,22}
\end{array}\right], & \widehat{\delta A_{1}}=\left[\begin{array}{cc}
\frac{(1-\epsilon)}{2} \delta a_{1,11} & -\epsilon \delta a_{1,12} \\
\delta a_{1,12} & \frac{(1-\epsilon)}{2} \delta a_{1,22}
\end{array}\right], \\
\delta D_{0}=\epsilon \delta D_{0}^{T}, & \delta D_{1}=-\epsilon \delta D_{1}^{T}, & \delta B_{j}=\left[\begin{array}{ll}
b_{j 1} & b_{j 2}
\end{array}\right],
\end{array}
$$

for $j=0,1$, and $U \in \mathbb{C}^{n \times n}$ being a unitary matrix such that $U=\left[\begin{array}{ll}V_{1} & V_{2}\end{array}\right]$ with $V_{1}=$ $\left[\begin{array}{ll}x_{1} & x_{2}\end{array}\right] \in \mathbb{C}^{n \times 2}$. Similar to Theorem 5.4, we get the following equations

$$
\begin{array}{rlrl}
\frac{(1+\epsilon)}{2} \lambda_{0} \delta a_{0, i i}+\frac{(1-\epsilon)}{2} \lambda_{1} \delta a_{1, i i} & =x_{i}^{T} k_{i}, & & i=1,2, \\
\lambda_{0} b_{0 i}+\lambda_{1} b_{1 i} & =V_{2}^{T} k_{i}, & i=1,2, \\
{\left[\begin{array}{cc}
\lambda_{0} & -\lambda_{1} \\
\lambda_{0} & \lambda_{1}
\end{array}\right]\left[\begin{array}{l}
\delta a_{0,12} \\
\delta a_{1,12}
\end{array}\right]} & =\left[\begin{array}{c}
x_{2}^{T} k_{1} \\
\epsilon x_{1}^{T} k_{2}
\end{array}\right] .
\end{array}
$$


The minimum norm solution of (7.2) is given by $b_{0 i}=\frac{\bar{\lambda}_{0}}{H_{2}^{2}(\lambda)} V_{2}^{T} k_{i}$ and $b_{1 i}=\frac{\bar{\lambda}_{1}}{H_{2}^{2}(\lambda)} V_{2}^{T} k_{i}$.

Case 1: When $\lambda_{0} \neq 0$ and $\lambda_{1} \neq 0$, the minimum norm solution of (7.1) is given by $\delta a_{0, i i}=\frac{(1+\epsilon)}{2} \frac{\bar{\lambda}_{0}}{G_{\epsilon}^{2}(\lambda)} x_{i}^{T} k_{i}$ and $\delta a_{1, i i}=\frac{(1-\epsilon)}{2} \frac{\bar{\lambda}_{1}}{G_{\epsilon}^{2}(\lambda)} x_{i}^{T} k_{i}$. In this case $\delta a_{0,12}=\frac{x_{2}^{T} k_{1}+\epsilon x_{1}^{T} k_{2}}{2 \lambda_{0}}$ and $\delta a_{1,12}=\frac{x_{2}^{T} k_{1}-\epsilon x_{1}^{T} k_{2}}{2 \lambda_{1}}$.

Case 2: When $\lambda_{0}=0$ but $\lambda_{1} \neq 0$, we find that the system (7.3) is consistent by using Remark 7.3. The minimum norm solution of (7.3) is given by $\delta a_{0,12}=0$ and $\delta a_{1,12}=\frac{x_{2}^{T} k_{1}}{\lambda_{1}}$. In this case $\delta a_{0, i i}=0$ and $\delta a_{1, i i}=-\frac{(1-\epsilon)}{2} x_{i}^{T} A_{1} x_{i}$. When $\lambda_{0} \neq 0$ but $\lambda_{1}=0$, we have that the system (7.3) is consistent by using Remark 7.3. The minimum norm solution of (7.3) is given by $\delta a_{0,12}=\frac{x_{2}^{T} k_{1}}{\lambda_{0}}$ and $\delta a_{1,12}=0$. In this case $\delta a_{0, i i}=-\frac{(1+\epsilon)}{2} x_{i}^{T} A_{0} x_{i}$ and $\delta a_{1, i i}=0$. Similar to earlier sections, we can get the backward error expression and perturbation matrices in each case.

REMARK 7.6. Suppose that $\left(\mu, x_{1}\right)$ and $\left(\mu, x_{2}\right)$ are two approximate eigenpairs of a nonhomogeneous $T$-even $/ T$-odd matrix pencil $\mathbf{L}$, where $\mu \in \mathbb{C}$ is a double-semisimple eigenvalue and $x_{1}, x_{2} \in \mathbb{C}^{n}$ are orthonormal vectors. Then similar to Corollary 3.7, by substituting $\lambda_{0}=1, \lambda_{1}=\mu$ in Theorem 7.5 and using [1, Theorem 3.4 ], we get

$$
\begin{aligned}
\left(\eta_{F}^{\mathbf{S}}\left(\mu, x_{1}, x_{2}, \mathbf{L}\right)\right)^{2} & =\left(\left(\eta_{F}^{\mathbf{S}}\left(\mu, x_{1}, \mathbf{L}\right)\right)^{2}+\left(\eta_{F}^{\mathbf{S}}\left(\mu, x_{2}, \mathbf{L}\right)\right)^{2}\right) \\
& =2\left(\left(\eta_{F}\left(\mu, x_{1}, \mathbf{L}\right)\right)^{2}+\left(\eta_{F}\left(\mu, x_{2}, \mathbf{L}\right)\right)^{2}\right),
\end{aligned}
$$

when $|\mu|=1$. Further, using Corollary 4.4, we get

$$
\eta_{F}^{\mathbf{S}}\left(\mu, x_{1}, x_{2}, \mathbf{L}\right)=\sqrt{2} \eta_{F}\left(\mu, x_{1}, x_{2}, \mathbf{L}\right),
$$

when $|\mu|=1$.

Finally, we summarize the relations between unstructured and structured backward errors of a single approximate eigenpair and two approximate eigenpairs for non-homogeneous matrix pencils. Let $\left(\mu, x_{1}\right)$ and $\left(\mu, x_{2}\right)$ be two approximate eigenpairs such that $\mu \in \mathbb{C}$ is a double-semisimple eigenvalue of a non-homogeneous matrix pencil $\mathbf{L}$. Then in Table 7.1, we present relationships between the structured backward error of two approximate eigenpairs of a double-semisimple eigenvalue $\eta_{F}^{\mathbf{S}}\left(\mu, x_{1}, x_{2}, \mathbf{L}\right)$, the unstructured backward error of two approximate eigenpairs of a double-semisimple eigenvalue $\eta_{F}\left(\mu, x_{1}, x_{2}, \mathbf{L}\right)$, and the structured backward error of a single approximate eigenpair $\eta_{F}^{\mathbf{S}}\left(\mu, x_{i}, \mathbf{L}\right)$, for $i=1,2$.

8. Numerical example. In this section, we illustrate our developed theory with a numerical example using Matlab 7.11.0. Let $\mathbf{L}$ be a T-skew-symmetric non-homogeneous $\left(\alpha_{0}=1\right)$ matrix pencil of the form (2.1). Let $A_{0}, A_{1}$ be defined by

$$
\begin{aligned}
A_{0} & =\left[\begin{array}{cccc}
0 & -0.2600+0.6487 i & -0.1135+0.3416 i & -0.3040-0.6366 i \\
0.2600-0.6487 i & 0 & -0.0914-0.5687 i & -0.7628+0.4553 i \\
0.1135-0.3416 i & 0.0914+0.5687 i & 0 & 0.3138-0.3496 i \\
0.3040+0.6366 i & 0.7628-0.4553 i & -0.3138+0.3496 i & 0
\end{array}\right], \\
A_{1} & =\left[\begin{array}{cccc}
0 & -0.0996-0.8100 i & 0.6837+0.2671 i & 0.0716+0.0580 i \\
0.0996+0.8100 i & 0 & 0.2214-0.5972 i & -0.2433-0.0032 i \\
-0.6837-0.2671 i & -0.2214+0.5972 i & 0 & 0.2821+0.2661 i \\
-0.0716-0.0580 i & 0.2433+0.0032 i & -0.2821-0.2661 i & 0
\end{array}\right] .
\end{aligned}
$$

These are random matrices satisfying $A_{0}=-A_{0}^{T}$ and $A_{1}=-A_{1}^{T}$. Clearly $\mathbf{L}$ is a regular matrix pencil. The approximate eigenpairs of $\mathbf{L}$ are obtained by using the Matlab command $[V, D]=\operatorname{eig}\left(A_{0}, A_{1}\right)$. Let $\mu=-D(2,2)=-D(3,3)$ be an approximate multiple eigenvalue of $\mathbf{L}$ with its corresponding eigenvectors given by $V(:, 2)$ and $V(:, 3)$. The orthonormal 
TABLE 7.1

Relations of backward errors for various cases

\begin{tabular}{|c|c|c|c|}
\hline Structure $(\mathbf{S})$ & $\begin{array}{l}\text { Relation between } \\
\eta_{F}^{\mathbf{S}}\left(\mu, x_{1}, x_{2}, \mathbf{L}\right) \boldsymbol{\&} \\
\eta_{F}^{\mathbf{S}}\left(\mu, x_{i}, \mathbf{L}\right), i=1,2\end{array}$ & $\begin{array}{l}\text { Relation between } \\
\eta_{F}^{\mathbf{S}}\left(\mu, x_{1}, x_{2}, \mathbf{L}\right) \mathbf{\&} \\
\eta_{F}\left(\mu, x_{1}, x_{2}, \mathbf{L}\right)\end{array}$ & $\begin{array}{l}\text { Relation between } \\
\eta_{F}^{\mathbf{S}}\left(\mu, x_{1}, x_{2}, \mathbf{L}\right) \mathbf{\&} \\
\eta_{F}\left(\mu, x_{i}, \mathbf{L}\right), i=1,2\end{array}$ \\
\hline $\begin{array}{l}T \text {-sym./ } \\
\text { T-sk.-sym. }\end{array}$ & $\begin{array}{l}\eta_{F}^{\mathbf{S}}\left(\mu, x_{1}, x_{2}, \mathbf{L}\right) \leq \\
\sqrt{\eta_{F}^{\mathbf{S}}\left(\mu, x_{1}, \mathbf{L}\right)^{2}+\eta_{F}^{\mathbf{S}}\left(\mu, x_{2}, \mathbf{L}\right)^{2}}\end{array}$ & $\begin{array}{l}\eta_{F}^{\mathbf{S}}\left(\mu, x_{1}, x_{2}, \mathbf{L}\right) \leq \\
\sqrt{2} \eta_{F}\left(\mu, x_{1}, x_{2}, \mathbf{L}\right)\end{array}$ & $\begin{array}{l}\eta_{F}^{\mathbf{S}}\left(\mu, x_{1}, x_{2}, \mathbf{L}\right) \leq \\
\sqrt{2} \sqrt{\eta_{F}^{2}\left(\mu, x_{1}, \mathbf{L}\right)+\eta_{F}^{2}\left(\mu, x_{2}, \mathbf{L}\right)}\end{array}$ \\
\hline $\begin{array}{l}\text { Herm./ } \\
\text { sk.-Herm. }\end{array}$ & $\begin{array}{l}\eta_{F}^{\mathbf{S}}\left(\mu, x_{1}, x_{2}, \mathbf{L}\right)= \\
\sqrt{\eta_{F}^{\mathbf{S}}\left(\mu, x_{1}, \mathbf{L}\right)^{2}+\eta_{F}^{\mathbf{S}}\left(\mu, x_{2}, \mathbf{L}\right)^{2}} \\
\text { for } \mu^{2}=-1\end{array}$ & $\begin{array}{l}\eta_{F}^{\mathbf{S}}\left(\mu, x_{1}, x_{2}, \mathbf{L}\right)= \\
\sqrt{2} \eta_{F}\left(\mu, x_{1}, x_{2}, \mathbf{L}\right) \\
\text { for } \mu^{2}=-1\end{array}$ & $\begin{array}{l}\eta_{F}^{\mathbf{S}}\left(\mu, x_{1}, x_{2}, \mathbf{L}\right)= \\
\sqrt{2} \sqrt{\eta_{F}^{2}\left(\mu, x_{1}, \mathbf{L}\right)+\eta_{F}^{2}\left(\mu, x_{2}, \mathbf{L}\right)} \\
\text { for } \mu^{2}=-1\end{array}$ \\
\hline $\begin{array}{l}\text { T-even/ } \\
T \text {-odd }\end{array}$ & $\begin{array}{l}\eta_{F}^{\mathbf{S}}\left(\mu, x_{1}, x_{2}, \mathbf{L}\right)= \\
\sqrt{\eta_{F}^{\mathbf{S}}\left(\mu, x_{1}, \mathbf{L}\right)^{2}+\eta_{F}^{\mathbf{S}}\left(\mu, x_{2}, \mathbf{L}\right)^{2}} \\
\text { for }|\mu|=1\end{array}$ & $\begin{array}{l}\eta_{F}^{\mathbf{S}}\left(\mu, x_{1}, x_{2}, \mathbf{L}\right)= \\
\sqrt{2} \eta_{F}\left(\mu, x_{1}, x_{2}, \mathbf{L}\right) \\
\text { for }|\mu|=1\end{array}$ & $\begin{array}{l}\eta_{F}^{\mathbf{S}}\left(\mu, x_{1}, x_{2}, \mathbf{L}\right)= \\
\sqrt{2} \sqrt{\eta_{F}^{2}\left(\mu, x_{1}, \mathbf{L}\right)+\eta_{F}^{2}\left(\mu, x_{2}, \mathbf{L}\right)} \\
\text { for }|\mu|=1\end{array}$ \\
\hline $\begin{array}{l}\text { H-even/ } \\
H \text {-odd }\end{array}$ & $\begin{array}{l}\eta_{F}^{\mathbf{S}}\left(\mu, x_{1}, x_{2}, \mathbf{L}\right)= \\
\sqrt{\eta_{F}^{\mathbf{S}}\left(\mu, x_{1}, \mathbf{L}\right)^{2}+\eta_{F}^{\mathbf{S}}\left(\mu, x_{2}, \mathbf{L}\right)^{2}} \\
\text { for } \mu^{2}=1\end{array}$ & $\begin{array}{l}\eta_{F}^{\mathbf{S}}\left(\mu, x_{1}, x_{2}, \mathbf{L}\right)= \\
\sqrt{2} \eta_{F}\left(\mu, x_{1}, x_{2}, \mathbf{L}\right) \\
\text { for } \mu^{2}=1\end{array}$ & $\begin{array}{l}\eta_{F}^{\mathbf{S}}\left(\mu, x_{1}, x_{2}, \mathbf{L}\right)= \\
\sqrt{2} \sqrt{\eta_{F}^{2}\left(\mu, x_{1}, \mathbf{L}\right)+\eta_{F}^{2}\left(\mu, x_{2}, \mathbf{L}\right)} \\
\text { for } \mu^{2}=1\end{array}$ \\
\hline
\end{tabular}

eigenvectors $x_{1}, x_{2}$ corresponding to $\mu$, are obtained by $x_{1}:=V(:, 2) /\|V(:, 2)\|$ and $x_{2}:=$ $(V(:, 3)-\gamma * V(:, 2)) /\|V(:, 3)-\gamma * V(:, 2)\|$, where $\gamma=\frac{V(:, 2)^{H} V(:, 3)}{V(:, 2)^{H} V(:, 2)}$. Using Theorem 3.3 for $\epsilon=-1$, we get the following perturbation matrices

$$
\begin{aligned}
\delta A_{0} & =\left[\begin{array}{cccc}
0 & -0.0170-0.4873 i & 0.3412-0.1463 i & 0.1048+0.2769 i \\
0.0170+0.4873 i & 0 & -0.0294-0.0085 i & 0.2473-0.0822 i \\
-0.3412+0.1463 i & 0.0294+0.0085 i & 0 & -0.0096+0.1557 i \\
-0.1048-0.2769 i & -0.2473+0.0822 i & 0.0096-0.1557 i & 0
\end{array}\right], \\
\delta A_{1} & =\left[\begin{array}{cccc}
0 & -0.0891+0.7143 i & -0.5315+0.1335 i & -0.0880-0.4281 i \\
0.0891-0.7143 i & -0 & 0.0409+0.0193 i & -0.3797+0.0621 i \\
0.5315-0.1335 i & -0.0409-0.0193 i & 0 & 0.0503-0.2247 i \\
0.0880+0.4281 i & 0.3797-0.0621 i & -0.0503+0.2247 i & 0
\end{array}\right] .
\end{aligned}
$$

We obtain $\eta_{F}^{\mathbf{S}}\left(\mu, x_{1}, x_{2}, \mathbf{L}\right)=1.8809$. Clearly $\mathbf{L}(\mu) x_{i}+\delta \mathbf{L}(\mu) x_{i}=0$, for $i=1,2$.

REMARK 8.1. When we encounter with two approximate eigenpairs $\left(\lambda, x_{1}\right)$ and $\left(\lambda, x_{2}\right)$, where $\lambda$ is a double-semisimple eigenvalue, the existing backward error theory of a single eigenpair fails to provide the minimum norm $\delta \mathbf{L} \in \mathbf{L}\left(\mathbb{C}^{n \times n}\right)$, which satisfies $(\mathbf{L}(\lambda)+$ $\delta \mathbf{L}(\lambda)) x_{i}=0$, for $i=1,2$. On the other hand, by using our theory, one can easily construct the required perturbed matrix pencil and backward error corresponding to two approximate eigenpairs of a double-semisimple eigenvalue.

9. Conclusions. We have studied the structured and unstructured backward error analysis of two approximate eigenpairs of a double-semisimple eigenvalue. We have investigated structured backward perturbations of eight special classes of structured matrix pencils, including T-symmetric, T-skew-symmetric, Hermitian, skew-Hermitian, T-even, T-odd, $H$-even, and $H$-odd cases. For each of those structures, we have obtained the minimal structured perturbed matrix pencil, with respect to the Frobenius norm such that the given two approximate eigenpairs become exact eigenpairs of an appropriately perturbed matrix pencil. We have also established the unstructured and structured backward error relationships between a single approximate eigenpair and two approximate eigenpairs of a double-semisimple eigenvalue. 
Acknowledgement. Thanks to the referees for their valuable suggestions of improving this manuscript.

\section{REFERENCES}

[1] B. ADHIKARI AND R. AlAM, Structured backward errors and pseudospectra of structured matrix pencils, SIAM J. Matrix Anal. Appl., 31 (2009), pp. 331-359.

[2] S. S. AHMAD, Perturbation analysis for palindromic and anti-palindromic nonlinear eigenvalue problems, Electron. Trans. Numer. Anal., 51 (2019), pp. 151-168. http://etna.ricam.oeaw.ac.at/vol.51.2019/pp151-168.dir/pp151-168.pdf

[3] S. S. AHMAD AND R. Alam, On Wilkinson's problem for matrix pencils, Electron. J. Linear Algebra, 30 (2015), pp. 632-648.

[4] S. S. Ahmad And V. Mehrmann, Perturbation analysis for complex symmetric, skew symmetric, even and odd matrix polynomials, Electron. Trans. Numer Anal., 38 (2011), pp. 275-302. http://etna.ricam.oeaw.ac.at/vol.38.2011/pp275-302.dir/pp275-302.pdf

[5] - Backward errors for eigenvalues and eigenvectors of Hermitian, skew-Hermitian, $H$-even and $H$-odd matrix polynomials, Linear Multilinear Algebra, 61 (2013), pp. 1244-1266.

[6] S. Bora, M. KAROw, C. MeHL, AND P. Sharma Structured eigenvalue backward errors of matrix pencils and polynomials with palindromic structures, SIAM J. Matrix Anal. Appl., 36 (2015), pp. 393-416.

[7] D. CHU AND G. H. GoluB, On a generalized eigenvalue problem for non-square matrix pencils, SIAM J. Matrix Anal. Appl., 28 (2006), pp. 770-787.

[8] M. GRACIA, Nearest matrix with two prescribed eigenvalues, Linear Algebra Appl., 401 (2005), pp. 277-294.

[9] S. GRaillat, A note on structured pseudospectra, J. Comput. Appl. Math., 191 (2006), pp. 68-76.

[10] E. KokABIFAR, G. B. Loghmani, AND S. M. Karbassi, Nearest matrix with prescribed eigenvalues and its applications, J. Comput. Appl. Math., 298 (2016), pp. 53-63.

[11] E. KoKabifar, G. B. Loghmani, P. J. Psarrakos, AND S. M. Karbassi, On the distance from a matrix polynomial to matrix polynomials with $k$ prescribed distinct eigenvalues, Linear Multilinear Algebra, 65 (2017), pp. 658-676.

[12] D. KRESSNER, Numerical Methods for General and Structured Eigenvalue Problems, Springer, Berlin, 2005.

[13] R. A. LIPPERT, Fixing two eigenvalues by a minimal perturbation, Linear Algebra Appl., 406 (2005), pp. 177200.

[14] _ Fixing multiple eigenvalues by a minimal perturbation, Linear Algebra Appl., 432 (2010), pp. 17851817.

[15] A. N. MALYShEV, A formula for the 2-norm distance from a matrix to the set of matrices with multiple eigenvalues, Numer. Math., 83 (1999), pp. 443-454.

[16] A. MuhiČ AND B. PlestenJaK, A method for computing all values $\lambda$ such that $A+\lambda B$ has a multiple eigenvalue, Linear Algebra Appl., 440 (2014), pp. 345-359.

[17] A. M. NAZARI AND D. RAJABI, Computational aspect to the nearest matrix with two prescribed eigenvalues, Linear Algebra Appl., 432 (2010), pp. 1-4.

[18] S. M. RUMP, Eigenvalues, pseudospectrum and structured perturbations, Linear Algebra Appl., 413 (2006), pp. 567-593.

[19] G. W. Stewart and J. G. Sun, Matrix Perturbation Theory, Academic Press, Boston, 1990.

[20] J. G. SUN, Backward perturbation analysis of certain characteristic subspaces, Numer. Math., 65 (1993), pp. 357-382.

[21] F. TISSEUR, A chart of backward errors for singly and doubly structured eigenvalue problems, SIAM J. Matrix Anal. Appl., 24 (2003), pp. 877-897.

[22] H. XIE, Sensitivity analysis of semi-simple eigenvalues of regular quadratic eigenvalue problems, Acta Math. Appl. Sin. Engl. Ser., 31 (2015), pp. 499-518.

[23] H. XIE AND H. DAI, On the sensitivity of multiple eigenvalues of nonsymmetric matrix pencils, Linear Algebra Appl., 374 (2003), pp. 143-158.

[24] K. Zhang AND Y. SU, Structured backward error analysis for sparse polynomial eigenvalue problems, Appl. Math. Comput., 219 (2012), pp. 3073-3082. 